Bull. Fac.Agric.,Cairo Univ., 64: 172-185 (2013).

\title{
EFFECT OF NITROGEN SOURCES ON GROWTH AND CHEMICAL CONSTITUENTS OF Cupressus sempervirens SEEDLINGS GROWN UNDER DIFFERENT LEVELS OF FIELD CAPACITY
}

(Received: 27.3.2013)

\author{
By \\ S. H. El-Hanafy, H. M. Swefy, A. R. Rabie* and E. H. M. Soliman* \\ Ornamental Horticulture Department, Faculty of Agriculture, Cairo University, \\ * Forestry Department, Horticulture Research Institute, Giza, Egypt
}

\begin{abstract}
This investigation was conducted at the Nursery of the Forestry Department, Horticulture Research Institute, Agriculture Research Center, Giza, Egypt and the Faculty of Agriculture, Cairo University, during two successive seasons of 2008/2009 and 2009/2010.

The objective of this investigation was to study the influence of three nitrogen fertilizer sources with different levels of field capacity on the growth and chemical constituents of Cupressus sempervirens seedlings. grown under different levels of field capacity. The chemical fertilizers used were ammonium sulfate $(\mathrm{NH} 4)_{2} \mathrm{SO}_{4}(20.5 \% \mathrm{~N})$, calcium nitrate $\mathrm{Ca}\left(\mathrm{NO}_{3}\right)_{2}(15.5 \% \mathrm{~N})$ and ammonium nitrate $\left(\mathrm{NH}_{4}\right)\left(\mathrm{NO}_{3}\right)_{2}$ $(33.5 \% \mathrm{~N})$ at the rates of $0.5,1.0,1.5$ and $2.0 \mathrm{~g} . \mathrm{N} /$ seedling with field capacity at levels of 60,80 and $100 \%$.

The results showed that, using calcium nitrate gave the highest values of stem length, stem diameter, fresh and dry weight of stem and leaves, in comparison with other nitrogen sources. In general, the application of three nitrogen sources in different rates resulted in increasing of $\mathrm{N}, \mathrm{P}$ and $\mathrm{K}$ leaf content as compared with the control. The highest content of proline in the leaves was obtained from of $2.0 \mathrm{~g}$. N/seedling with $60 \%$ field capacity level. Whereas, calcium nitrate decreased proline content in comparison with the other nitrogen sources under different field capacity levels.
\end{abstract}

Key words: ammonium nitrate, ammonium sulfate, calcium nitrate, Cupressus sempervirens, nitrogen fertilizer and water deficit.

\section{INTRODUCTION}

Cupressus sempervirens belongs to family Cupressaceae which has been distributed throughout the Mediterranean region since classical times. In its native soil, grows in a tapering columnar arrived to a height of $90 \mathrm{ft}$. Its branches are thickly covered with small, imbricate, shiny green leaves. The timer is hard, dose-grained, and of a fine reddish hue and very durable. Cupressus sempervirens has a gloomy and forbidding but wonderfully stately aspect (Aromatherapy Global Online Research Archive,2000).

Nitrogen is one of the essential elements in plant nutrition. Lack of adequate nitrogen subsequently produces plants that are lighter green in color due to a smaller amount of chlorophyll (Follett, 1981). Several works have been done on the effect of nitrogen, Sannappa et al. (2000) observed that leaf yield of Mulberry had maximum values under the application of calcium ammonium nitrate in comparison with ammonium chloride and ammonium sulfate. Warren and Adams (2002) on Pinus pinaster, found that, dry mass was unaffected by $\mathrm{N}$ form at 0.125 or $0.5 \mathrm{mM} \mathrm{N}$. In contrast, dry mass of seedlings supplied with ammonium or ammonium nitrate at 2.0 and $8.0 \mathrm{mM} \mathrm{N}$, was approximately threefold greater than seedlings supplied with nitrate alone. Rance et al. (2009) used $\mathrm{N}$ fertilizer labelled with ${ }^{15} \mathrm{~N}$ to follow the accumulation and distribution of $\mathrm{N}$ applied at different times after planting Eucalyptus grandis seedlings. Results revealed that, after 1 year aboveground biomass of the controls was only $30 \%$ of the fertilized trees. At later applications, controls were not significantly different from fertilized trees up to 
1 year later. Zhao and Liu (2009) identified the short-term influences of experimental warming, nitrogen fertilization, and their combination on growth and physiological performances of Picea asperata seedlings. Results illustrated that nitrogen fertilization significantly improved plant growth in unwarmed plots, by stimulating total biomass, maximum net photosynthetic rate $(A \max )$, antioxidant compounds. However, in warmed plots, nitrogen addition clearly decreased $A$ max, antioxidant compounds. Andivia et al. (2012) found in holm oak Quercus ilex, a very small increment in $\mathrm{N}$ doses during the autumn (1.5 vs. $0.0 \mathrm{mg} \mathrm{N})$ improved some morphological parameters, such as stem diameter (D) and shoot dry weight, and physiological parameters, such as total antioxidant activity. Siemens and Zwiazek (2013) studied the effects of high concentrations $(4,8$, or $16 \mathrm{mM})$ of nitrate $\left(\mathrm{NO}_{3}\right)$ and ammonium $\left(\mathrm{NH}_{4}\right)$ on water relations and growth of trembling aspen seedlings in solution culture. Results demonstrated that, aspen seedlings are tolerant of high nitrate concentrations, but intolerant of high ammonium concentrations. Ammonium was not toxic to aspen seedlings at moderate concentrations and that the seedlings were capable of assimilating and utilizing both ammonium and nitrate as a nitrogen source.

Water is the most limiting ecological resource for most tree and forest sites. As soil-water content declines, trees become more stressed and begin to react to resource availability changes (Coder, 1999). The growing concerns about water scarcity have focused more attention on water management in agriculture and promotion of water conservation through improved water use efficiency (WUE) (Bacelar et al., 2012). In this respect, Xiao et al. (2009) subjected Populus cathayana plantlets to continuous drought stress by withholding soil water content at $25 \%$ of field capacity (FC) for 45 days, while the control treatments were kept at $100 \%$ FC. Results revealed that, drought stress significantly inhibited plant growth, decreased net photosynthetic rate and stomatal conductance of leaves. Zheng et al. (2010) found that, as water stress increased, stomatal conductance $\left(g_{\mathrm{s}}\right)$, net photosynthetic rate $\left(\begin{array}{ll}P_{\mathrm{N}}\end{array}\right)$, transpiration rate $(E)$, and leaf RWC decreased while LMA increased in all provenances. Results illustrated that dry mass was reduced in droughted plants and the percentage increased in dry mass allocated to roots. Yang et al. (2011) exposed Acer mono seedlings to two watering regimes (well watered (100\% of field capacity) and drought (30\% of field capacity)).Results illustrated that drought significantly reduced growth and gas exchange characteristics of $A$. mono, including net photosynthetic rate $\left(P_{\mathrm{N}}\right)$, stomatal conductance $(g$ s). Eldhuset et al. (2013) reported how an 11-week drought affected tracheid structure and above- and belowground growth in 5-year-old Norway spruce trees (Picea abies) under controlled conditions. Results demonstrated that the canopy of trees subjected to severe drought had significantly less current-year needle biomass, and fewer tracheids and tracheid rows in current-year shoots compared to fully watered control trees. Li et al. (2013) exposed Tamarix ramosissima and Populus euphratica to different groundwater treatments: inundation, drought, and relatively shallow, moderate and deep groundwater. Results showed that, under inundation, $T$. ramosissima showed little growth whereas $P$. euphratica died after 45 days. Droughted seedlings of both species suffered from considerable water stress evidenced by slow growth, decreased total leaf area and specific leaf area, and decreased xylem water potential $(\psi)$, maximum photosynthetic rate and carboxylation efficiency.

The translocation of chemical signals within plants is important for plant adaptation to stress, especially abiotic stresses such as drought (Goodger and Schachtman, 2010).

This study aimed to investigate how nitrogen decreases the harmful effects of water deficit using three sources of nitrogen (ammonium sulphate, calcium nitrate and ammonium nitrate) with three levels of field capacity (60- $80-100 \%)$.

\section{MATERIALS AND METHODS}

The present investigation was conducted at the Nursery of the Forestry Department, Horticulture Research Institute, Agriculture Research Center, Giza, Egypt and the Faculty of Agriculture, Cairo University, Giza, Egypt, during two successive seasons of 2008-2009 and 2009-2010.

One-year-old seedlings of Cupressus sempervirens obtained from the nursery of the Forestry Department, Horticulture Research Institute, Agriculture Research Center, Giza, Egypt, were planted on the second week of April in both seasons in $25 \mathrm{~cm}$ diameter plastic pots, filled with $7.5 \mathrm{Kg}$ of sandy soil and remained in pots to the second week of April of 2009 and 2010, respectively. 
Seedlings were $30-35 \mathrm{~cm}$ in height and 0.3- 0.4 $\mathrm{cm}$ in stem diameter. Each pot contained only one healthy seedling. Experiment consisted of 39 treatments, 3 fertilizer sources, 4 fertilizer concentrations for every source and 3 levels of field capacity in addition to three control treatments (60$80-100 \%$ of water field capacity without fertilizer).

The chemical fertilizers and levels of soil moisture used in this study were:

Ammonium sulfate (NH4)2 SO4 $(20.5 \% \mathrm{~N})$

$\mathrm{N} 1=0.5 \mathrm{~g}$. nitrogen $=2.4 \mathrm{~g}$. fertilizer

$\mathrm{N} 2=1.0$ g. nitrogen $=4.8$ g. fertilizer

$\mathrm{N} 3=1.5 \mathrm{~g}$. nitrogen $=7.2 \mathrm{~g}$. fertilizer

$\mathrm{N} 4=2.0 \mathrm{~g}$. nitrogen $=9.6 \mathrm{~g}$. fertilizer

Calcium nitrate $\mathrm{Ca}(\mathrm{NO} 3) 2(15.5 \% \mathrm{~N})$

$\mathrm{N} 1=0.5 \mathrm{~g}$. nitrogen $=3.2 \mathrm{~g}$. fertilizer

$\mathrm{N} 2=1.0 \mathrm{~g}$. nitrogen $=6.4 \mathrm{~g}$. fertilizer

$\mathrm{N} 3=1.5 \mathrm{~g}$. nitrogen $=9.6 \mathrm{~g}$. fertilizer

$\mathrm{N} 4=2.0 \mathrm{~g}$. nitrogen $=12.8 \mathrm{~g}$. fertilizer

Ammonium nitrate (NH4) (NO3)2 (33.5\% N)

$\mathrm{N} 1=0.5 \mathrm{~g}$. nitrogen $=1.5 \mathrm{~g}$. fertilizer

$\mathrm{N} 2=1.0 \mathrm{~g}$. nitrogen $=3.0 \mathrm{~g}$. fertilizer

$\mathrm{N} 3=1.5 \mathrm{~g}$. nitrogen $=4.5 \mathrm{~g}$. fertilizer

$\mathrm{N} 4=2.0$ g. nitrogen $=6.0$ g. fertilizer

Levels of soil moisture treatments:

$60 \%$ of field capacity $=0.50 \mathrm{~L}$ of water

$80 \%$ of field capacity $=0.70 \mathrm{~L}$ of water

$100 \%$ of field capacity $=0.85 \mathrm{~L}$ of water

After two months from transplanting, the fertilizers were applied separately every 3 months until the end of the experiment in April of 2009 and 2010, respectively. Fertilized and non fertilized plants were irrigated at 60,80 and 100\% water field capacity. Irrigation was applied once weekly in winter and twice weekly in summer.

\section{The following data were recorded Vegetative growth}

The vegetative growth parameter: Stem length, stem fresh and dry weight, leaf fresh and dry weight, root fresh and dry weight were detemined. The dry weight of the samples was determined after drying the samples in an oven at $70^{\circ} \mathrm{C}$ till a constant weight.

\section{Chemical constituents}

The chemical analysis was carried out on leaf samples obtained from all treatments. Determination of $\mathrm{N} \%, \mathrm{P} \%, \mathrm{~K} \%$ and proline $(\mathrm{mg} / \mathrm{gm})$ in leaves was acheived.

\section{Mineral determination}

Dry samples of leaves $(0.2 \mathrm{gm}$. each $)$ were used to determine total soluble nitrogen $(\mathrm{N})$, phosphorus (P), Potassium (K) and proline according to Pregl, (1945), Piper, (1947), Brown and Lililand (1946), and Bates et al. (1973), respectively.

\section{Statistical analyses}

The layout of the experiment was a completely randomized block design in factorial arrangement. The differences between the means of the treatments for the experiment were compared by using LSD at $5 \%$ probability, according to Snedecor and Cochran (1972).

\section{Soil physical analysis}

The physical analysis of the used soil was done according to the pipette method as described by Black (1965) (Table, A).

\section{Soil and water analysis}

For soil, a (1:5 extract) was used for determination of elements. The soil was shaken with

Table (A): Soil physical analysis

\begin{tabular}{|l|l|l|l|l|l|l|}
\hline Sample & Coarse sand (\%) & Fine sand (\%) & Clay (\%) & Silt (\%) & $\begin{array}{l}\text { CaCo } \\
3\end{array}$ & Texture class \\
\hline & 38.88 & 42.50 & 5.20 & 7.10 & 5.02 & Sandy \\
\hline
\end{tabular}

Table (B): Soil chemical analysis

\begin{tabular}{|l|l|l|l|l|l|l|l|l|l|}
\hline Sample & $\mathrm{pH}$ & $\begin{array}{l}\text { E.C. } \\
(\mathrm{mm} \text { hos } \mid \mathrm{cm})\end{array}$ & \multicolumn{3}{l|}{ Soluble anions and cations (meq/l) } & \multicolumn{2}{l|}{ Mineral elements (ppm) } \\
\hline & 7.5 & 1.00 & $\mathrm{Cl}$ & $\mathrm{SO} 4$ & $\mathrm{Ca}$ & $\mathrm{Na}$ & $\mathrm{K}$ & $\mathrm{N}$ & $\mathrm{P}$ \\
\cline { 3 - 9 } & & 0.95 & 0.70 & 0.75 & 1.30 & 0.21 & 7.00 & 1.3 \\
\hline
\end{tabular}

Table (C): Water chemical analysis

\begin{tabular}{|l|l|l|l|l|l|l|l|l|}
\hline Sample & $\begin{array}{l}\text { E.C. } \\
(\mathrm{mm} \text { hos } \mid \mathrm{cm})\end{array}$ & \multicolumn{3}{l|}{ Soluble anions and cations (meq/l) } & \multicolumn{2}{l|}{ Mineral Elements (mg/L) } \\
\hline \multirow{2}{*}{1.05} & $\mathrm{Cl}$ & $\mathrm{SO} 4$ & $\mathrm{Ca}$ & $\mathrm{Na}$ & $\mathrm{K}$ & $\mathrm{N}$ & $\mathrm{P}$ \\
\cline { 3 - 9 } & 1.08 & 2.44 & 3.00 & 6.02 & 0.20 & 1.50 & 0.14 \\
\hline
\end{tabular}


$100 \mathrm{ml}$ deionised water per $20 \mathrm{~g}$ soil. The chemical analysis of the used soil was performed before the experiment according to the methods using in plant (Table, B). For water, $100 \mathrm{ml}$ were taken for determing the elements. (Table, C).

\subsection{Vegetative growth \\ 3.1.1. Stem length}

Data presented in Table (1) show that, using calcium nitrate at the rates of $1.5(\mathrm{~N} 3)$ and $2.0 \mathrm{~g} \mathrm{~N}$ (N4) resulted in the highest values of stem length $(80.98$ and $83.36 \mathrm{~cm})$ and $(83.50$ and $86.06 \mathrm{~cm})$ in the first and the second seasons, respectively regardless of field capacity level.

The lowest values of stem length were obtained with $60 \%$ of field capacity levels regardless of fertilizer source or its rate in comparison with 80 or $100 \%$ of field capacity in both seasons.

The highest value of stem length resulted from using calcium nitrate at the rate of $2.0 \mathrm{~g}$. N/seedling with $100 \%$ of field capacity level in comparison with other treatments in both seasons.

\subsubsection{Stem diameter}

Data presented in Table (2) illustrate that, the application of calcium nitrate or ammonium sulfate at the rate of $2.0 \mathrm{~g} \mathrm{~N}(\mathrm{~N} 4)$, regardless of field capacity level, gave the thickest value of stem diameter in the first season. In the second one, the rate of $0.5,1,1.5$ and $2.0 \mathrm{~g} \mathrm{~N} /$ seedling gave the highest values of stem diameter regardless of field capacity level, with application of calcium nitrate.

The highest values of stem diameter were obtained with $100 \%$ of field capacity level in comparison with 60 or $80 \%$ of field capacity in the first and the second seasons, respectively, regardless of fertilizer source or its rate.

The highest value of stem diameter resulted from using calcium nitrate at the rate of $2.0 \mathrm{~g}$ $\mathrm{N} /$ seedling with $100 \%$ of water field capacity level in comparison with the other treatments in the first season. In the second one, the highest values of stem diameter were obtained from 1, 2.0g $\mathrm{N} /$ seedling with $100 \%$ field capacity level.

\subsubsection{Stem fresh weight}

Data presented in Table (3) indicated that, using of $2.0 \mathrm{~g} \mathrm{~N} /$ seedling of calcium nitrate gave the heaviest stem fresh weight (40.78 and $43.32 \mathrm{~g}$. /seedling) in the first and second seasons, respectively, regardless of field capacity level.
Using $60 \%$ of water field capacity level resulted in the lowest values of stem fresh weight in comparison with 80 or $100 \%$ of field capacity, respectively in both seasons, regardless of fertilizer source or its rate.

The highest values of stem fresh weight resulted from using calcium nitrate at the rate of $1.5 \mathrm{~g} \mathrm{~N}$ with $100 \%$ field capacity level and $2.0 \mathrm{~g} \mathrm{~N} /$ seedling with 80 and $100 \%$ of field capacity levels in both seasons.

\subsubsection{Stem dry weight}

Data presented in Table (4) illustrate that, adding nitrogen at the rate of $2.0 \mathrm{~g} \mathrm{~N} /$ seedling from calcium nitrate gave the heaviest values of stem dry weight (19.50 and 20.73g. /seedling) in the first and the second seasons, respectively regardless of field capacity level.

The heaviest stem dry weights resulted from calcium nitrate at the rate of $2.0 \mathrm{~g} \mathrm{~N} / \mathrm{seedling}$ with $80 \%$ and $100 \%$ of field capacity levels in both seasons.

\subsubsection{Leaves fresh weight}

Data in Table (5) showed that, using nitrogen at the rate of $2.0 \mathrm{~g} \mathrm{~N} /$ seedling from calcium nitrate gave the heaviest values of leaves fresh weight (70.26 and 69.96g. /seedling) in the first and the second seasons, respectively regardless of field capacity level.

Using $100 \%$ field capacity level gave the highest values of stem fresh weight in comparison with 60 or $80 \%$ field capacity levels in the first and the second seasons, respectively, regardless of fertilizer source or its rate.

In the first season, application of ammonium sulfate and calcium nitrate at the rate of $1.5 \mathrm{~g} \mathrm{~N}$ under $100 \%$ of water field capacity level and the same fertilizers at the rate of $2.0 \mathrm{~g} \mathrm{~N}$ under 80 and $100 \%$ of field capacity levels resulted in the highest values of leaves fresh weight. In the second season, the highest values were obtained by using calcium nitrate at the rate of $2.0 \mathrm{~g} \mathrm{~N}$ under $100 \%$ of water field capacity level.

\subsubsection{Leaves dry weight}

Data presented in Table (6) show that, using both calcium nitrate and ammonium sulfate at the rate of $2.0 \mathrm{~g} \mathrm{~N} / \mathrm{seedling}$ gave the highest values of leaves dry weight in the first and the second seasons, respectively regardless of field capacity levels.

The highest values of leaf dry weight were obtained by using calcium nitrate at the rate of $2.0 \mathrm{~g}$ 
Table (1): Effect of nitrogen sources and rates under different levels of water field capacity on stem length (cm.) of Cupressus sempervirens seedlings during the seasons of 2008-2009 and 2009-2010.

\begin{tabular}{|c|c|c|c|c|c|c|c|c|c|c|c|c|}
\hline \multicolumn{13}{|c|}{ First season } \\
\hline Fertilizer & \multicolumn{4}{|c|}{ Calcium nitrate } & \multicolumn{4}{|c|}{ Ammonium sulfate } & \multicolumn{4}{|c|}{ Ammonium nitrate } \\
\hline & $\begin{array}{l}60 \% \\
\text { F.C. }\end{array}$ & $\begin{array}{l}80 \% \\
\text { F.C. }\end{array}$ & $\begin{array}{c}100 \% \\
\text { F.C. }\end{array}$ & Mean & $\begin{array}{l}60 \% \\
\text { F.C. }\end{array}$ & $\begin{array}{l}80 \% \\
\text { F.C. }\end{array}$ & $\begin{array}{l}100 \% \\
\text { F.C. }\end{array}$ & Mean & $\begin{array}{l}60 \% \\
\text { F.C. }\end{array}$ & $\begin{array}{l}\text { 80\% } \\
\text { F.C. }\end{array}$ & $\begin{array}{c}100 \% \\
\text { F.C. }\end{array}$ & Mean \\
\hline $\begin{array}{l}\text { control } \\
\text { (zero) }\end{array}$ & $\begin{array}{c}50.53 \\
O\end{array}$ & $\begin{array}{c}52.90 \\
\text { NO }\end{array}$ & $\begin{array}{c}54.40 \\
\mathrm{NO}\end{array}$ & $\begin{array}{c}52.61 \\
G\end{array}$ & $\begin{array}{c}50.53 \\
O\end{array}$ & $\begin{array}{c}52.90 \\
\text { NO }\end{array}$ & $\begin{array}{c}54.40 \\
\text { NO }\end{array}$ & $\begin{array}{c}52.61 \\
\mathrm{G}\end{array}$ & $\begin{array}{c}50.53 \\
O\end{array}$ & $\begin{array}{c}52.90 \\
\text { NO }\end{array}$ & $\begin{array}{c}54.40 \\
\text { NO }\end{array}$ & $\begin{array}{c}52.61 \\
\mathrm{G}\end{array}$ \\
\hline N1(0.5g.) & $\begin{array}{c}57.67 \\
\mathrm{~L}-\mathrm{O}\end{array}$ & $\begin{array}{c}57.77 \\
\mathrm{~L}-\mathrm{O}\end{array}$ & $\begin{array}{c}68.73 \\
\text { F-I }\end{array}$ & $\begin{array}{l}61.39 \\
\text { D-F }\end{array}$ & $\begin{array}{c}53.73 \\
\text { NO }\end{array}$ & $\begin{array}{c}59.90 \\
\text { J-N }\end{array}$ & $\begin{array}{c}66.20 \\
\text { G-K }\end{array}$ & $\begin{array}{c}59.94 \\
\text { EF }\end{array}$ & $\begin{array}{c}50.83 \\
O\end{array}$ & $\begin{array}{c}60.30 \\
\text { I-N }\end{array}$ & $\begin{array}{c}66.80 \\
\text { G-K }\end{array}$ & $\begin{array}{c}59.31 \\
F\end{array}$ \\
\hline N2(1g.) & $\begin{array}{l}62.90 \\
\mathrm{H}-\mathrm{M}\end{array}$ & $\begin{array}{c}67.57 \\
\text { G-J }\end{array}$ & $\begin{array}{c}98.60 \\
\text { B }\end{array}$ & $\begin{array}{c}76.36 \\
\text { B }\end{array}$ & $\begin{array}{l}57.42 \\
\mathrm{M}-\mathrm{O}\end{array}$ & $\begin{array}{c}65.97 \\
\text { G-L }\end{array}$ & $\begin{array}{c}71.17 \\
\text { E-H }\end{array}$ & $\begin{array}{c}64.84 \\
\text { CD }\end{array}$ & $\begin{array}{c}53.73 \\
\text { NO }\end{array}$ & $\begin{array}{c}61.33 \\
\text { I-N }\end{array}$ & $\begin{array}{l}76.60 \\
D-F\end{array}$ & $\begin{array}{c}63.89 \\
\text { C-E }\end{array}$ \\
\hline N3(1.5g.) & $\begin{array}{c}59.03 \\
\text { K-O }\end{array}$ & $\begin{array}{c}81.63 \\
\text { CD }\end{array}$ & $\begin{array}{c}102.3 \\
\text { B }\end{array}$ & $\begin{array}{c}80.98 \\
\text { A }\end{array}$ & $\begin{array}{c}70.80 \\
\text { E-H }\end{array}$ & $\begin{array}{l}71.53 \\
\text { E-G }\end{array}$ & $\begin{array}{c}85.20 \\
C\end{array}$ & $\begin{array}{c}75.84 \\
\text { B }\end{array}$ & $\begin{array}{c}64.10 \\
\text { G-M }\end{array}$ & $\begin{array}{c}66.17 \\
\text { G-K }\end{array}$ & $\begin{array}{c}68.10 \\
\text { G-J }\end{array}$ & $\begin{array}{c}66.12 \\
C\end{array}$ \\
\hline N4(2g.) & $\begin{array}{l}58.43 \\
\mathrm{~K}-\mathrm{O}\end{array}$ & $\begin{array}{c}79.83 \\
\text { CD }\end{array}$ & $\begin{array}{c}111.8 \\
\mathrm{~A}\end{array}$ & $\begin{array}{c}83.36 \\
\text { A }\end{array}$ & $\begin{array}{l}56.73 \\
\mathrm{M}-\mathrm{O}\end{array}$ & $\begin{array}{c}70.47 \\
\text { E-H }\end{array}$ & $\begin{array}{l}77.20 \\
\text { DE }\end{array}$ & $\begin{array}{c}68.13 \\
C\end{array}$ & $\begin{array}{c}27.48 \\
P\end{array}$ & $\begin{array}{c}61.20 \\
\text { I-N }\end{array}$ & $\begin{array}{c}67.43 \\
\text { G-J }\end{array}$ & $\begin{array}{c}52.03 \\
\mathrm{G}\end{array}$ \\
\hline Mean & $\begin{array}{c}57.71 \\
\mathrm{E} \\
\end{array}$ & $\begin{array}{c}67.94 \\
\text { BC } \\
\end{array}$ & $\begin{array}{c}87.16 \\
\mathrm{~A}\end{array}$ & & $\begin{array}{c}57.70 \\
\mathrm{E} \\
\end{array}$ & $\begin{array}{c}64.15 \\
\mathrm{D} \\
\end{array}$ & $\begin{array}{c}70.83 \\
\text { B } \\
\end{array}$ & & $\begin{array}{c}49.42 \\
\mathrm{~F} \\
\end{array}$ & $\begin{array}{c}60.38 \\
\mathrm{E} \\
\end{array}$ & $\begin{array}{c}66.67 \\
\text { CD } \\
\end{array}$ & \\
\hline \multicolumn{13}{|c|}{ Second season } \\
\hline Fertilizer & \multicolumn{4}{|c|}{ Calcium nitrate } & \multicolumn{4}{|c|}{ Ammonium sulfate } & \multicolumn{4}{|c|}{ Ammonium nitrate } \\
\hline & $\begin{array}{l}60 \% \\
\text { F.C. }\end{array}$ & $\begin{array}{l}80 \% \\
\text { F.C. }\end{array}$ & $\begin{array}{c}100 \% \\
\text { F.C. }\end{array}$ & Mean & $\begin{array}{l}60 \% \\
\text { F.C. }\end{array}$ & $\begin{array}{l}80 \% \\
\text { F.C. }\end{array}$ & $\begin{array}{l}100 \% \\
\text { F.C. }\end{array}$ & Mean & $\begin{array}{l}60 \% \\
\text { F.C. }\end{array}$ & $\begin{array}{l}80 \% \\
\text { F.C. }\end{array}$ & $\begin{array}{c}100 \% \\
\text { F.C. }\end{array}$ & Mean \\
\hline $\begin{array}{l}\text { control } \\
\text { (zero) }\end{array}$ & $\begin{array}{c}51.83 \\
\mathrm{Q}\end{array}$ & $\begin{array}{c}51.87 \\
Q\end{array}$ & $\begin{array}{l}59.27 \\
\text { M-Q }\end{array}$ & $\begin{array}{c}54.32 \\
\mathrm{G}\end{array}$ & $\begin{array}{c}51.83 \\
Q\end{array}$ & $\begin{array}{c}51.87 \\
Q\end{array}$ & $\begin{array}{c}59.27 \\
\text { M-Q }\end{array}$ & $\begin{array}{c}54.32 \\
\mathrm{G}\end{array}$ & $\begin{array}{c}51.83 \\
\mathrm{Q}\end{array}$ & $\begin{array}{c}51.87 \\
Q\end{array}$ & $\begin{array}{l}59.27 \\
\text { M-Q }\end{array}$ & $\begin{array}{c}54.32 \\
\mathrm{G}\end{array}$ \\
\hline N1(0.5g.) & $\begin{array}{l}59.50 \\
M-Q\end{array}$ & $\begin{array}{c}58.10 \\
\text { N-Q }\end{array}$ & $\begin{array}{c}72.23 \\
\text { F-H }\end{array}$ & $\begin{array}{c}63.28 \\
\mathrm{EF}\end{array}$ & $\begin{array}{c}54.70 \\
\text { O-Q }\end{array}$ & $\begin{array}{c}61.97 \\
\mathrm{I}-\mathrm{O}\end{array}$ & $\begin{array}{c}68.63 \\
\mathrm{H}-\mathrm{K}\end{array}$ & $\begin{array}{c}61.77 \\
F\end{array}$ & $\begin{array}{c}52.17 \\
\text { PQ }\end{array}$ & $\begin{array}{c}60.43 \\
\text { K-Q }\end{array}$ & $\begin{array}{c}68.17 \\
\text { H-L }\end{array}$ & $\begin{array}{c}60.26 \\
F\end{array}$ \\
\hline N2(1g.) & $\begin{array}{c}61.03 \\
\mathrm{~J}-\mathrm{O}\end{array}$ & $\begin{array}{c}69.93 \\
\text { G-I }\end{array}$ & $\begin{array}{c}101.6 \\
\text { B }\end{array}$ & $\begin{array}{c}77.53 \\
\text { B }\end{array}$ & $\begin{array}{l}58.97 \\
M-Q\end{array}$ & $\begin{array}{c}66.73 \\
\text { H-N }\end{array}$ & $\begin{array}{c}74.03 \\
\text { E-H }\end{array}$ & $\begin{array}{c}66.58 \\
\text { DE }\end{array}$ & $\begin{array}{c}54.23 \\
\mathrm{O}-\mathrm{Q}\end{array}$ & $\begin{array}{c}62.23 \\
\mathrm{I}-\mathrm{O}\end{array}$ & $\begin{array}{c}77.70 \\
\text { D-G }\end{array}$ & $\begin{array}{c}64.72 \\
\mathrm{EF}\end{array}$ \\
\hline N3(1.5g.) & $\begin{array}{c}65.63 \\
\text { K-P }\end{array}$ & $\begin{array}{c}83.77 \\
\text { CD }\end{array}$ & $\begin{array}{c}106.1 \\
\text { B }\end{array}$ & $\begin{array}{c}85.10 \\
\text { A }\end{array}$ & $\begin{array}{c}72.63 \\
\text { F-H }\end{array}$ & $\begin{array}{c}72.60 \\
\text { F-H }\end{array}$ & $\begin{array}{c}86.70 \\
\text { C }\end{array}$ & $\begin{array}{c}77.31 \\
\text { B }\end{array}$ & $\begin{array}{c}65.50 \\
\mathrm{H}-\mathrm{N}\end{array}$ & $\begin{array}{l}67.03 \\
\text { H-M }\end{array}$ & $\begin{array}{c}78.60 \\
\text { D-F }\end{array}$ & $\begin{array}{c}64.72 \\
\mathrm{EF}\end{array}$ \\
\hline N4(2g.) & $\begin{array}{c}59.73 \\
\text { L-Q }\end{array}$ & $\begin{array}{c}83.27 \\
\text { CD }\end{array}$ & $\begin{array}{c}115.2 \\
\text { A }\end{array}$ & $\begin{array}{c}86.06 \\
\text { A }\end{array}$ & $\begin{array}{l}59.43 \\
M-Q\end{array}$ & $\begin{array}{c}72.97 \\
\text { F-H }\end{array}$ & $\begin{array}{c}81.57 \\
\text { C-E }\end{array}$ & $\begin{array}{c}71.32 \\
C\end{array}$ & $\begin{array}{c}30.57 \\
\text { R }\end{array}$ & $\begin{array}{c}69.40 \\
\text { H-J }\end{array}$ & $\begin{array}{c}78.40 \\
\mathrm{D}-\mathrm{F}\end{array}$ & $\begin{array}{c}59.45 \\
F\end{array}$ \\
\hline Mean & $\begin{array}{c}59.55 \\
F\end{array}$ & $\begin{array}{c}69.39 \\
\mathrm{C}\end{array}$ & $\begin{array}{c}90.88 \\
\text { A }\end{array}$ & & $\begin{array}{c}59.51 \\
\mathrm{EF}\end{array}$ & $\begin{array}{c}65.23 \\
\mathrm{D}\end{array}$ & $\begin{array}{c}74.04 \\
\text { B }\end{array}$ & & $\begin{array}{c}50.86 \\
\mathrm{G}\end{array}$ & $\begin{array}{c}62.19 \\
\mathrm{DE}\end{array}$ & $\begin{array}{c}72.43 \\
\text { BC }\end{array}$ & \\
\hline
\end{tabular}

Means followed by a letter in common in the same column are not significantly different at 0.05 level of probability 
Table (2): Effect of nitrogen sources and rates under different levels of water field capacity on stem diameter (cm.) of Cupressus sempervirens seedlings during the seasons of 2008-2009 and 2009-2010.

\begin{tabular}{|c|c|c|c|c|c|c|c|c|c|c|c|c|}
\hline \multicolumn{13}{|c|}{ First season } \\
\hline Fertilizer & \multicolumn{4}{|c|}{ Calcium nitrate } & \multicolumn{4}{|c|}{ Ammonium sulfate } & \multicolumn{4}{|c|}{ Ammonium nitrate } \\
\hline & $\begin{array}{l}60 \% \\
\text { F.C. }\end{array}$ & $\begin{array}{r}80 \% \\
\text { F.C. }\end{array}$ & $\begin{array}{c}100 \% \\
\text { F.C. }\end{array}$ & Mean & $\begin{array}{l}60 \% \\
\text { F.C. }\end{array}$ & $\begin{array}{l}80 \% \\
\text { F.C. }\end{array}$ & $\begin{array}{l}100 \% \\
\text { F.C. }\end{array}$ & Mean & $\begin{array}{l}60 \% \\
\text { F.C. }\end{array}$ & $\begin{array}{l}80 \% \\
\text { F.C. }\end{array}$ & $\begin{array}{c}100 \% \\
\text { F.C. }\end{array}$ & Mean \\
\hline $\begin{array}{l}\text { control } \\
\text { (zero) }\end{array}$ & $\begin{array}{c}0.40 \\
\mathrm{~L}\end{array}$ & $\begin{array}{c}0.46 \\
\mathrm{~L}\end{array}$ & $\begin{array}{c}0.60 \\
\mathrm{~K}\end{array}$ & $\begin{array}{c}0.48 \\
\mathrm{~F} \\
\end{array}$ & $\begin{array}{c}0.40 \\
\mathrm{~L}\end{array}$ & $\begin{array}{c}0.46 \\
\mathrm{~L}\end{array}$ & $\begin{array}{c}0.60 \\
\mathrm{~K} \\
\end{array}$ & $0.48 \mathrm{~F}$ & $\begin{array}{c}0.40 \\
\mathrm{~L}\end{array}$ & $\begin{array}{c}0.46 \\
\mathrm{~L}\end{array}$ & $\begin{array}{c}0.60 \\
\mathrm{~K}\end{array}$ & $0.48 \mathrm{~F}$ \\
\hline N1(0.5g.) & $\begin{array}{c}0.76 \\
\text { F-J } \\
\end{array}$ & $\begin{array}{l}0.83 \\
\text { E-H } \\
\end{array}$ & $\begin{array}{c}0.80 \\
\text { E-I } \\
\end{array}$ & $\begin{array}{c}0.80 \\
\mathrm{CD} \\
\end{array}$ & $\begin{array}{c}0.60 \\
\mathrm{~K} \\
\end{array}$ & $\begin{array}{c}0.63 \\
\text { JK } \\
\end{array}$ & $\begin{array}{l}0.70 \\
\mathrm{H}-\mathrm{K} \\
\end{array}$ & $0.64 \mathrm{E}$ & $\begin{array}{c}0.66 \\
\mathrm{I}-\mathrm{K} \\
\end{array}$ & $\begin{array}{c}0.76 \\
\text { F-J } \\
\end{array}$ & $\begin{array}{l}0.83 \\
\text { E-H } \\
\end{array}$ & $0.75 \mathrm{D}$ \\
\hline N2(1g.) & $\begin{array}{c}0.76 \\
\text { F-J } \\
\end{array}$ & $\begin{array}{l}0.96 \\
\text { A-D } \\
\end{array}$ & $\begin{array}{l}0.96 \\
\text { A-D } \\
\end{array}$ & $\begin{array}{c}0.90 \\
\mathrm{AB} \\
\end{array}$ & $\begin{array}{c}0.76 \\
\text { F-J } \\
\end{array}$ & $\begin{array}{l}0.86 \\
\mathrm{D}-\mathrm{G} \\
\end{array}$ & $\begin{array}{l}0.90 \\
\mathrm{C}-\mathrm{F} \\
\end{array}$ & $0.84 \mathrm{BC}$ & $\begin{array}{l}0.70 \\
\mathrm{H}-\mathrm{K} \\
\end{array}$ & $\begin{array}{l}0.73 \\
\text { G-K } \\
\end{array}$ & $\begin{array}{c}0.80 \\
\text { E-I } \\
\end{array}$ & $0.74 \mathrm{D}$ \\
\hline N3(1.5g.) & $\begin{array}{c}0.76 \\
\text { F-J } \\
\end{array}$ & $\begin{array}{l}0.83 \\
\text { E-H } \\
\end{array}$ & $\begin{array}{c}0.80 \\
\text { E-I }\end{array}$ & $\begin{array}{l}0.80 \\
\mathrm{CD} \\
\end{array}$ & $\begin{array}{l}0.70 \\
\mathrm{H}-\mathrm{K} \\
\end{array}$ & $\begin{array}{l}0.83 \\
\text { E-H } \\
\end{array}$ & $\begin{array}{l}0.90 \\
\mathrm{C}-\mathrm{F} \\
\end{array}$ & $0.81 \mathrm{CD}$ & $\begin{array}{c}0.60 \\
\mathrm{~K} \\
\end{array}$ & $\begin{array}{l}0.70 \\
\mathrm{H}-\mathrm{K} \\
\end{array}$ & $\begin{array}{l}0.73 \\
\text { G-K } \\
\end{array}$ & $0.67 \mathrm{E}$ \\
\hline N4(2g.) & $\begin{array}{l}0.83 \\
\text { E-H }\end{array}$ & $\begin{array}{l}0.93 \\
\text { B-E }\end{array}$ & $\begin{array}{c}1.03 \\
\mathrm{~A}\end{array}$ & $0.93 \mathrm{~A}$ & $\begin{array}{c}0.80 \\
\text { E-I }\end{array}$ & $\begin{array}{l}1.0 \\
\mathrm{~A}-\mathrm{C}\end{array}$ & $\begin{array}{c}1.0 \\
\text { A -C }\end{array}$ & $0.95 \mathrm{~A}$ & $\begin{array}{l}0.33 \\
\text { L-M }\end{array}$ & $\begin{array}{c}0.63 \\
\text { JK }\end{array}$ & $\begin{array}{c}0.66 \\
\mathrm{I}-\mathrm{K}\end{array}$ & $0.53 \mathrm{~F}$ \\
\hline Mean & $\begin{array}{l}0.70 \\
\mathrm{CD} \\
\end{array}$ & $\begin{array}{c}0.80 \\
\mathrm{AB} \\
\end{array}$ & $\begin{array}{c}0.84 \\
\mathrm{~A} \\
\end{array}$ & & $\begin{array}{c}0.65 \\
\mathrm{D} \\
\end{array}$ & $\begin{array}{c}0.76 \\
\mathrm{BC} \\
\end{array}$ & $\begin{array}{c}0.83 \\
\mathrm{~A} \\
\end{array}$ & & $\begin{array}{c}0.54 \\
\mathrm{E} \\
\end{array}$ & $\begin{array}{c}0.66 \\
\mathrm{D} \\
\end{array}$ & $\begin{array}{c}0.72 \\
\mathrm{C} \\
\end{array}$ & \\
\hline \multicolumn{13}{|c|}{ Second season } \\
\hline Fertilizer & \multicolumn{4}{|c|}{ Calcium nitrate } & \multicolumn{4}{|c|}{ Ammonium sulfate } & \multicolumn{4}{|c|}{ Ammonium nitrate } \\
\hline & $\begin{array}{l}60 \% \\
\text { F.C. }\end{array}$ & $\begin{array}{l}80 \% \\
\text { F.C. }\end{array}$ & $\begin{array}{c}100 \% \\
\text { F.C. }\end{array}$ & Mean & $\begin{array}{l}60 \% \\
\text { F.C. }\end{array}$ & $\begin{array}{l}80 \% \\
\text { F.C. }\end{array}$ & $\begin{array}{l}100 \% \\
\text { F.C. }\end{array}$ & Mean & $\begin{array}{l}60 \% \\
\text { F.C. }\end{array}$ & $\begin{array}{l}80 \% \\
\text { F.C. }\end{array}$ & $\begin{array}{c}100 \% \\
\text { F.C. }\end{array}$ & Mean \\
\hline $\begin{array}{l}\text { control } \\
\text { (zero) }\end{array}$ & $\begin{array}{c}0.43 \\
\mathrm{~J} \\
\end{array}$ & $\begin{array}{c}0.50 \\
\mathrm{IJ} \\
\end{array}$ & $\begin{array}{c}0.56 \\
\text { H-J }\end{array}$ & $\begin{array}{c}0.50 \\
\mathrm{E} \\
\end{array}$ & $\begin{array}{c}0.43 \\
\mathrm{~J} \\
\end{array}$ & $\begin{array}{c}0.50 \\
\text { IJ }\end{array}$ & $\begin{array}{c}0.56 \\
\text { H-J } \\
\end{array}$ & $0.50 \quad \mathrm{E}$ & $\begin{array}{c}0.43 \\
\mathrm{~J} \\
\end{array}$ & $\begin{array}{c}0.50 \\
\mathrm{IJ} \\
\end{array}$ & $\begin{array}{c}0.56 \\
\text { HIJ }\end{array}$ & $\begin{array}{c}0.50 \\
\mathrm{E} \\
\end{array}$ \\
\hline N1(0.5g.) & $\begin{array}{l}0.76 \\
\mathrm{C}-\mathrm{F}\end{array}$ & $\begin{array}{l}0.86 \\
\mathrm{~A}-\mathrm{C}\end{array}$ & $\begin{array}{l}0.76 \\
\mathrm{C}-\mathrm{F}\end{array}$ & $0.80 \mathrm{AB}$ & $\begin{array}{c}0.63 \\
\text { F-I }\end{array}$ & $\begin{array}{l}0.70 \\
\mathrm{D}-\mathrm{H}\end{array}$ & $\begin{array}{l}0.73 \\
\mathrm{C}-\mathrm{G}\end{array}$ & $\begin{array}{c}0.68 \\
\mathrm{CD}\end{array}$ & $\begin{array}{l}0.70 \\
\text { D-H }\end{array}$ & $\begin{array}{l}0.70 \\
\mathrm{D}-\mathrm{H}\end{array}$ & $\begin{array}{l}0.73 \\
\text { C-G }\end{array}$ & $\begin{array}{c}0.71 \\
\mathrm{CD}\end{array}$ \\
\hline N2(1g.) & $\begin{array}{l}0.83 \\
\text { A-D }\end{array}$ & $\begin{array}{l}0.76 \\
\mathrm{C}-\mathrm{F}\end{array}$ & $\begin{array}{c}0.96 \\
\mathrm{~A}\end{array}$ & $0.85 \mathrm{~A}$ & $\begin{array}{c}0.60 \\
\text { G-I }\end{array}$ & $\begin{array}{l}0.76 \\
\mathrm{C}-\mathrm{F}\end{array}$ & $\begin{array}{l}0.76 \\
\mathrm{C}-\mathrm{F}\end{array}$ & $\begin{array}{c}0.71 \\
\mathrm{CD}\end{array}$ & $\begin{array}{l}0.66 \\
\text { E-H }\end{array}$ & $\begin{array}{l}0.73 \\
\mathrm{C}-\mathrm{G}\end{array}$ & $\begin{array}{l}0.70 \\
\text { D-H }\end{array}$ & $\begin{array}{l}0.70 \\
\mathrm{CD}\end{array}$ \\
\hline N3(1.5g.) & $\begin{array}{l}0.80 \\
\text { B-E }\end{array}$ & $\begin{array}{c}0.93 \\
\mathrm{AB}\end{array}$ & $\begin{array}{l}0.86 \\
\text { A-C }\end{array}$ & $0.86 \mathrm{~A}$ & $\begin{array}{l}0.73 \\
\text { C-G }\end{array}$ & $\begin{array}{l}0.80 \\
\text { B-E }\end{array}$ & $\begin{array}{l}0.76 \\
\mathrm{C}-\mathrm{F}\end{array}$ & $\begin{array}{c}0.76 \\
\mathrm{BC}\end{array}$ & $\begin{array}{c}0.60 \\
\text { G-I }\end{array}$ & $\begin{array}{c}0.63 \\
\text { F-I }\end{array}$ & $\begin{array}{l}0.66 \\
\text { E-H }\end{array}$ & $\begin{array}{c}0.63 \\
\mathrm{D}\end{array}$ \\
\hline N4(2g.) & $\begin{array}{l}0.76 \\
\mathrm{C}-\mathrm{F}\end{array}$ & $\begin{array}{l}0.83 \\
\text { A-D }\end{array}$ & $\begin{array}{c}0.96 \\
\mathrm{~A}\end{array}$ & $0.85 \mathrm{~A}$ & $\begin{array}{l}0.76 \\
\mathrm{C}-\mathrm{F}\end{array}$ & $\begin{array}{l}0.86 \\
\text { A-C }\end{array}$ & $\begin{array}{c}0.93 \\
\mathrm{AB}\end{array}$ & $0.85 \mathrm{~A}$ & $\begin{array}{c}0.33 \\
\mathrm{~J}\end{array}$ & $\begin{array}{c}0.63 \\
\text { F-I }\end{array}$ & $\begin{array}{l}0.70 \\
D-H\end{array}$ & $\begin{array}{c}0.55 \\
\mathrm{E}\end{array}$ \\
\hline Mean & $\begin{array}{l}0.72 \\
\mathrm{BC}\end{array}$ & $\begin{array}{c}0.78 \\
\mathrm{AB}\end{array}$ & $\begin{array}{c}0.82 \\
\text { A }\end{array}$ & & $\begin{array}{c}0.63 \\
\mathrm{D}\end{array}$ & $\begin{array}{l}0.72 \\
\mathrm{BC}\end{array}$ & $\begin{array}{c}0.75 \\
\text { B }\end{array}$ & & $\begin{array}{c}0.54 \\
\mathrm{E}\end{array}$ & $\begin{array}{c}0.64 \\
\mathrm{D}\end{array}$ & $\begin{array}{l}0.67 \\
C D\end{array}$ & \\
\hline
\end{tabular}

Means followed by a letter in common in the same column are not significantly different at 0.05 level of probability 
Table (3): Effect of nitrogen sources and rates under different levels of water field capacity on stem fresh weight (g./seedling) of Cupressus sempervirens seedlings during the seasons of 2008-2009 and 2009-2010.

\begin{tabular}{|c|c|c|c|c|c|c|c|c|c|c|c|c|}
\hline \multicolumn{13}{|c|}{ First season } \\
\hline Fertilizer & \multicolumn{4}{|c|}{ Calcium nitrate } & \multicolumn{4}{|c|}{ Ammonium sulfate } & \multicolumn{4}{|c|}{ Ammonium nitrate } \\
\hline & $\begin{array}{l}60 \% \\
\text { F.C. }\end{array}$ & $\begin{array}{l}80 \% \\
\text { F.C. }\end{array}$ & $\begin{array}{c}100 \% \\
\text { F.C. }\end{array}$ & Mean & $\begin{array}{l}60 \% \\
\text { F.C. }\end{array}$ & $\begin{array}{l}\text { 80\% } \\
\text { F.C. }\end{array}$ & $\begin{array}{l}100 \% \\
\text { F.C. }\end{array}$ & Mean & $\begin{array}{c}60 \% \\
\text { F.C. }\end{array}$ & $\begin{array}{l}\text { 80\% } \\
\text { F.C. }\end{array}$ & $\begin{array}{c}100 \% \\
\text { F.C. }\end{array}$ & Mean \\
\hline $\begin{array}{l}\text { control } \\
\text { (zero) }\end{array}$ & $\begin{array}{c}14.17 \\
\mathrm{O}\end{array}$ & $\begin{array}{c}21.80 \\
\text { K-N }\end{array}$ & $\begin{array}{l}20.03 \\
\text { M-O }\end{array}$ & $18.67 \mathrm{FG}$ & $\begin{array}{c}14.17 \\
\mathrm{O}\end{array}$ & $\begin{array}{c}21.80 \\
\text { K-N }\end{array}$ & $\begin{array}{l}20.03 \\
\mathrm{M}-\mathrm{O}\end{array}$ & 18.67FG & $\begin{array}{c}14.17 \\
\mathrm{O}\end{array}$ & $\begin{array}{c}21.80 \\
\text { K-N }\end{array}$ & $\begin{array}{l}20.03 \\
\text { M-O }\end{array}$ & $18.67 \mathrm{FG}$ \\
\hline N1(0.5g.) & $\begin{array}{l}18.47 \\
\mathrm{~N}-\mathrm{O}\end{array}$ & $\begin{array}{c}25.63 \\
\text { G-M }\end{array}$ & $\begin{array}{c}29.10 \\
\text { E-J }\end{array}$ & $24.40 \mathrm{E}$ & $\begin{array}{c}20.13 \\
\text { L-O }\end{array}$ & $\begin{array}{c}20.40 \\
\text { L-N }\end{array}$ & $\begin{array}{c}23.43 \\
\mathrm{~J}-\mathrm{N}\end{array}$ & $21.32 \mathrm{~F}$ & $\begin{array}{c}29.67 \\
\text { D-I }\end{array}$ & $\begin{array}{c}30.93 \\
\text { C-H }\end{array}$ & $\begin{array}{c}33.60 \\
\text { B-F }\end{array}$ & $\begin{array}{c}31.40 \\
\mathrm{C}\end{array}$ \\
\hline N2(1g.) & $\begin{array}{c}21.80 \\
\mathrm{~K}-\mathrm{N}\end{array}$ & $\begin{array}{c}26.33 \\
\text { G-L }\end{array}$ & $\begin{array}{c}34.97 \\
B-E\end{array}$ & $27.70 \mathrm{D}$ & $\begin{array}{c}20.73 \\
\text { L-N }\end{array}$ & $\begin{array}{l}25.03 \\
\text { H-M }\end{array}$ & $\begin{array}{c}28.17 \\
\text { F-J }\end{array}$ & 24.64DE & $\begin{array}{c}29.87 \\
\text { D-I }\end{array}$ & $\begin{array}{c}33.13 \\
\text { B-F }\end{array}$ & $\begin{array}{c}34.50 \\
\text { B-E }\end{array}$ & $\begin{array}{c}32.50 \\
\mathrm{C}\end{array}$ \\
\hline N3(1.5g.) & $\begin{array}{c}31.23 \\
C-G\end{array}$ & $\begin{array}{c}37.67 \\
\text { B }\end{array}$ & $\begin{array}{c}43.83 \\
\text { A }\end{array}$ & $37.58 \mathrm{~B}$ & $\begin{array}{c}25.50 \\
\text { G-M }\end{array}$ & $\begin{array}{c}34.70 \\
\text { B-E }\end{array}$ & $\begin{array}{c}35.80 \\
\text { B-D }\end{array}$ & $\begin{array}{c}32.00 \\
\mathrm{C}\end{array}$ & $\begin{array}{c}23.87 \\
\mathrm{I}-\mathrm{N}\end{array}$ & $\begin{array}{c}27.47 \\
\text { F-K }\end{array}$ & $\begin{array}{c}30.27 \\
\text { D-H }\end{array}$ & 27.20DE \\
\hline N4(2g.) & $\begin{array}{l}33.03 \\
\text { B-F }\end{array}$ & $\begin{array}{c}44.13 \\
\text { A }\end{array}$ & $\begin{array}{c}45.17 \\
\text { A }\end{array}$ & $40.78 \mathrm{~A}$ & $\begin{array}{c}33.50 \\
\text { B-F }\end{array}$ & $\begin{array}{c}36.93 \\
\text { BC }\end{array}$ & $\begin{array}{c}38.17 \\
\text { B }\end{array}$ & $\begin{array}{c}36.20 \\
\text { B }\end{array}$ & $\begin{array}{c}15.50 \\
\mathrm{O}\end{array}$ & $\begin{array}{c}23.43 \\
\mathrm{~J}-\mathrm{N}\end{array}$ & $\begin{array}{c}25.03 \\
\text { H-M }\end{array}$ & $21.32 \mathrm{~F}$ \\
\hline Mean & $\begin{array}{c}23.74 \\
\text { D }\end{array}$ & $\begin{array}{r}31.11 \\
\mathrm{~B} \\
\end{array}$ & $\begin{array}{r}34.62 \\
\mathrm{~A} \\
\end{array}$ & & $\begin{array}{c}22.81 \\
\mathrm{DE}\end{array}$ & $\begin{array}{r}27.11 \\
\mathrm{C} \\
\end{array}$ & $\begin{array}{c}29.12 \\
\mathrm{BC}\end{array}$ & & $\begin{array}{c}22.61 \\
\text { DE }\end{array}$ & $\begin{array}{c}26.69 \\
\mathrm{C} \\
\end{array}$ & $\begin{array}{r}28.69 \\
\mathrm{C} \\
\end{array}$ & \\
\hline \multicolumn{13}{|c|}{ Second season } \\
\hline Fertilizer & \multicolumn{4}{|c|}{ Calcium nitrate } & \multicolumn{4}{|c|}{ Ammonium sulfate } & \multicolumn{4}{|c|}{ Ammonium nitrate } \\
\hline & $\begin{array}{l}60 \% \\
\text { F.C. }\end{array}$ & $\begin{array}{l}80 \% \\
\text { F.C. }\end{array}$ & $\begin{array}{c}100 \% \\
\text { F.C. }\end{array}$ & Mean & $\begin{array}{l}60 \% \\
\text { F.C. }\end{array}$ & $\begin{array}{l}80 \% \\
\text { F.C. }\end{array}$ & $\begin{array}{l}100 \% \\
\text { F.C. }\end{array}$ & Mean & $\begin{array}{r}60 \% \\
\text { F.C. }\end{array}$ & $\begin{array}{l}80 \% \\
\text { F.C. }\end{array}$ & $\begin{array}{c}100 \% \\
\text { F.C. }\end{array}$ & Mean \\
\hline $\begin{array}{l}\text { control } \\
\text { (zero) }\end{array}$ & $\begin{array}{c}15.07 \\
P\end{array}$ & $\begin{array}{c}21.07 \\
\mathrm{O}\end{array}$ & $\begin{array}{c}21.67 \\
O\end{array}$ & $19.27 \mathrm{~K}$ & $\begin{array}{c}15.07 \\
P\end{array}$ & $\begin{array}{c}21.07 \\
\mathrm{O}\end{array}$ & $\begin{array}{c}21.67 \\
0\end{array}$ & $19.27 \mathrm{~K}$ & $\begin{array}{c}15.07 \\
P\end{array}$ & $\begin{array}{c}21.07 \\
\mathrm{O}\end{array}$ & $\begin{array}{c}21.67 \\
O\end{array}$ & $\begin{array}{c}19.27 \\
\mathrm{~K}\end{array}$ \\
\hline N1(0.5g.) & $\begin{array}{c}21.50 \\
\mathrm{O}\end{array}$ & $\begin{array}{c}24.70 \\
\mathrm{~K}-\mathrm{O}\end{array}$ & $\begin{array}{c}26.80 \\
\mathrm{I}-\mathrm{M}\end{array}$ & $24.33 \mathrm{IJ}$ & $\begin{array}{c}21.60 \\
O\end{array}$ & $\begin{array}{c}22.27 \\
\text { NO }\end{array}$ & $\begin{array}{c}25.07 \\
\text { K-O }\end{array}$ & $22.98 \mathrm{~J}$ & $\begin{array}{c}31.77 \\
\text { E-H }\end{array}$ & $\begin{array}{c}32.10 \\
\text { E-H }\end{array}$ & $\begin{array}{c}34.50 \\
\text { C-F }\end{array}$ & $\begin{array}{c}32.79 \\
\mathrm{DE}\end{array}$ \\
\hline N2(1g.) & $\begin{array}{l}23.47 \\
\mathrm{M}-\mathrm{O}\end{array}$ & $\begin{array}{c}27.83 \\
\text { H-L }\end{array}$ & $\begin{array}{c}29.53 \\
\text { G-J }\end{array}$ & $26.94 \mathrm{GH}$ & $\begin{array}{l}23.27 \\
\mathrm{M}-\mathrm{O}\end{array}$ & $\begin{array}{c}26.07 \\
\mathrm{~J}-\mathrm{N}\end{array}$ & $\begin{array}{c}29.43 \\
\text { G-J }\end{array}$ & $26.26 \mathrm{HI}$ & $\begin{array}{c}31.50 \\
\text { E-H }\end{array}$ & $\begin{array}{c}32.13 \\
\text { E-H }\end{array}$ & $\begin{array}{c}35.67 \\
\text { C-E }\end{array}$ & $\begin{array}{c}33.10 \\
\mathrm{D}\end{array}$ \\
\hline N3(1.5g.) & $\begin{array}{c}34.27 \\
\text { C-F }\end{array}$ & $\begin{array}{c}42.23 \\
\text { B }\end{array}$ & $\begin{array}{c}44.07 \\
\mathrm{AB}\end{array}$ & $40.19 \mathrm{~B}$ & $\begin{array}{c}28.17 \\
\text { H-K }\end{array}$ & $\begin{array}{c}30.80 \\
\text { F-I }\end{array}$ & $\begin{array}{c}33.57 \\
\text { D-G }\end{array}$ & $\begin{array}{c}30.84 \\
\text { EF }\end{array}$ & $\begin{array}{c}25.37 \\
\text { J-O }\end{array}$ & $\begin{array}{c}29.37 \\
\text { G-J }\end{array}$ & $\begin{array}{c}31.67 \\
\text { E-H }\end{array}$ & $\begin{array}{c}28.80 \\
\text { FG }\end{array}$ \\
\hline N4(2g.) & $\begin{array}{c}37.30 \\
\text { CD }\end{array}$ & $\begin{array}{c}45.37 \\
\mathrm{AB}\end{array}$ & $\begin{array}{c}47.30 \\
\text { A }\end{array}$ & $43.32 \mathrm{~A}$ & $\begin{array}{c}34.63 \\
\text { C-F }\end{array}$ & $\begin{array}{c}36.90 \\
\text { CD }\end{array}$ & $\begin{array}{c}38.20 \\
\mathrm{C}\end{array}$ & $\begin{array}{c}36.58 \\
\mathrm{C}\end{array}$ & $\begin{array}{c}16.06 \\
P\end{array}$ & $\begin{array}{c}23.93 \\
\text { K-O }\end{array}$ & $\begin{array}{c}23.83 \\
\text { L-O }\end{array}$ & $\begin{array}{c}21.27 \\
\mathrm{~J}\end{array}$ \\
\hline Mean & $\begin{array}{c}26.32 \\
D\end{array}$ & $\begin{array}{c}32.24 \\
\text { B }\end{array}$ & $\begin{array}{r}33.87 \\
\mathrm{~A}\end{array}$ & & $\begin{array}{c}24.55 \\
E\end{array}$ & $\begin{array}{c}27.42 \\
\text { D }\end{array}$ & $\begin{array}{c}29.59 \\
C\end{array}$ & & $\begin{array}{c}23.95 \\
\text { EF }\end{array}$ & $\begin{array}{c}27.72 \\
\text { D }\end{array}$ & $\begin{array}{c}29.47 \\
\mathrm{C}\end{array}$ & \\
\hline
\end{tabular}

Means followed by a letter in common in the same column are not significantly different at 0.05 level of probability 
Table (4): Effect of nitrogen sources and rates under different levels of water field capacity on stem dry weight (g./seedling) of Cupressus sempervirens seedlings during the seasons of 2008-2009 and 2009-2010.

\begin{tabular}{|c|c|c|c|c|c|c|c|c|c|c|c|c|}
\hline \multicolumn{13}{|c|}{ First season } \\
\hline Fertilizer & \multicolumn{4}{|c|}{ Calcium nitrate } & \multicolumn{4}{|c|}{ Ammonium sulfate } & \multicolumn{4}{|c|}{ Ammonium nitrate } \\
\hline & $\begin{array}{l}60 \% \\
\text { F.C. }\end{array}$ & $\begin{array}{l}80 \% \\
\text { F.C. }\end{array}$ & $\begin{array}{c}100 \% \\
\text { F.C. }\end{array}$ & Mean & $\begin{array}{l}60 \% \\
\text { F.C. }\end{array}$ & $\begin{array}{l}80 \% \\
\text { F.C. }\end{array}$ & $\begin{array}{l}100 \% \\
\text { F.C. }\end{array}$ & Mean & $\begin{array}{l}60 \% \\
\text { F.C. }\end{array}$ & $\begin{array}{l}80 \% \\
\text { F.C. }\end{array}$ & $\begin{array}{c}100 \% \\
\text { F.C. }\end{array}$ & Mean \\
\hline $\begin{array}{l}\text { control } \\
\text { (zero) }\end{array}$ & $\begin{array}{c}7.86 \\
\mathrm{~L}\end{array}$ & $\begin{array}{l}8.76 \\
\text { KL }\end{array}$ & $\begin{array}{c}11.03 \\
\mathrm{I}-\mathrm{K}\end{array}$ & $9.22 \mathrm{H}$ & $\begin{array}{c}7.86 \\
\mathrm{~L}\end{array}$ & $\begin{array}{l}8.76 \\
\text { KL }\end{array}$ & $\begin{array}{c}11.03 \\
\mathrm{I}-\mathrm{K}\end{array}$ & $9.22 \mathrm{H}$ & $\begin{array}{c}7.86 \\
\mathrm{~L}\end{array}$ & $\begin{array}{l}8.76 \\
\text { K-L }\end{array}$ & $\begin{array}{c}11.03 \\
\mathrm{I}-\mathrm{K}\end{array}$ & $9.22 \mathrm{H}$ \\
\hline N1(0.5g.) & $\begin{array}{l}9.36 \\
\text { KL }\end{array}$ & $\begin{array}{c}12.80 \\
\text { E-J }\end{array}$ & $\begin{array}{c}14.40 \\
\text { C-H }\end{array}$ & $12.19 \mathrm{~F}$ & $\begin{array}{c}10.37 \\
\mathrm{~J}-\mathrm{L}\end{array}$ & $\begin{array}{c}10.73 \\
\mathrm{I}-\mathrm{K}\end{array}$ & $\begin{array}{c}11.10 \\
\mathrm{I}-\mathrm{K}\end{array}$ & $10.73 \mathrm{G}$ & $\begin{array}{c}15.47 \\
B-E\end{array}$ & $\begin{array}{c}14.70 \\
\text { C-H }\end{array}$ & $\begin{array}{c}14.87 \\
\mathrm{C}-\mathrm{H}\end{array}$ & 15.01CD \\
\hline N2(1g.) & $\begin{array}{c}10.83 \\
\mathrm{I}-\mathrm{K}\end{array}$ & $\begin{array}{c}12.37 \\
\text { G-J }\end{array}$ & $\begin{array}{c}16.80 \\
\mathrm{BC}\end{array}$ & $13.33 \mathrm{EF}$ & $\begin{array}{c}10.43 \\
\mathrm{~J}-\mathrm{L}\end{array}$ & $\begin{array}{c}12.57 \\
\text { F-J }\end{array}$ & $\begin{array}{c}13.43 \\
\text { E-I }\end{array}$ & $12.14 \mathrm{~F}$ & $\begin{array}{c}13.97 \\
\mathrm{D}-\mathrm{H}\end{array}$ & $\begin{array}{c}15.50 \\
B-E\end{array}$ & $\begin{array}{l}15.13 \\
\text { B-F }\end{array}$ & $14.87 \mathrm{CD}$ \\
\hline N3(1.5g.) & $\begin{array}{c}15.00 \\
\mathrm{C}-\mathrm{G}\end{array}$ & $\begin{array}{c}17.67 \\
\text { B } \\
\end{array}$ & $\begin{array}{c}21.87 \\
\mathrm{~A} \\
\end{array}$ & $18.18 \mathrm{~B}$ & $\begin{array}{c}12.60 \\
\text { F-J }\end{array}$ & $\begin{array}{c}14.73 \\
\mathrm{C}-\mathrm{H} \\
\end{array}$ & $\begin{array}{c}15.47 \\
\text { B-E }\end{array}$ & $\begin{array}{c}14.27 \mathrm{C}- \\
\mathrm{E}\end{array}$ & $\begin{array}{c}12.20 \\
\text { H-J }\end{array}$ & $\begin{array}{c}14.03 \\
\mathrm{D}-\mathrm{H}\end{array}$ & $\begin{array}{c}14.57 \\
\mathrm{C}-\mathrm{H}\end{array}$ & 13.60DE \\
\hline N4(2g.) & $\begin{array}{c}14.83 \\
\text { C-H }\end{array}$ & $\begin{array}{c}21.10 \\
\mathrm{~A}\end{array}$ & $\begin{array}{c}22.57 \\
\text { A }\end{array}$ & $19.50 \mathrm{~A}$ & $\begin{array}{c}14.17 \\
\text { C-H }\end{array}$ & $\begin{array}{l}16.37 \\
\text { B-D }\end{array}$ & $\begin{array}{l}16.37 \\
\text { B-D }\end{array}$ & $15.63 \mathrm{C}$ & $\begin{array}{l}7.73 \\
\text { K-M }\end{array}$ & $\begin{array}{c}10.27 \\
\mathrm{~J}-\mathrm{L}\end{array}$ & $\begin{array}{c}11.23 \\
\mathrm{I}-\mathrm{K}\end{array}$ & $9.73 \mathrm{H}$ \\
\hline Mean & $\begin{array}{c}11.58 \\
\text { D }\end{array}$ & $\begin{array}{c}14.51 \\
\text { B } \\
\end{array}$ & $\begin{array}{c}17.33 \\
\mathrm{~A} \\
\end{array}$ & & $\begin{array}{c}11.09 \\
\mathrm{DE}\end{array}$ & $\begin{array}{c}12.63 \\
\mathrm{C} \\
\end{array}$ & $\begin{array}{c}13.48 \\
\mathrm{C} \\
\end{array}$ & & $\begin{array}{c}11.45 \\
\mathrm{DE}\end{array}$ & $\begin{array}{c}12.65 \\
\mathrm{C} \\
\end{array}$ & $\begin{array}{c}13.37 \\
\mathrm{C} \\
\end{array}$ & \\
\hline \multicolumn{13}{|c|}{ Second season } \\
\hline Fertilizer & \multicolumn{4}{|c|}{ Calcium nitrate } & \multicolumn{4}{|c|}{ Ammonium sulfate } & \multicolumn{4}{|c|}{ Ammonium nitrate } \\
\hline & $\begin{array}{l}60 \% \\
\text { F.C. }\end{array}$ & $\begin{array}{l}80 \% \\
\text { F.C. } \\
\end{array}$ & $\begin{array}{c}100 \% \\
\text { F.C. }\end{array}$ & Mean & $\begin{array}{l}60 \% \\
\text { F.C. }\end{array}$ & $\begin{array}{l}80 \% \\
\text { F.C. }\end{array}$ & $\begin{array}{l}100 \% \\
\text { F.C. }\end{array}$ & Mean & $\begin{array}{l}60 \% \\
\text { F.C. }\end{array}$ & $\begin{array}{l}80 \% \\
\text { F.C. }\end{array}$ & $\begin{array}{c}100 \% \\
\text { F.C. }\end{array}$ & Mean \\
\hline $\begin{array}{l}\text { control } \\
\text { (zero) }\end{array}$ & $\begin{array}{c}7.96 \\
P\end{array}$ & $\begin{array}{c}11.40 \\
\mathrm{~L}-\mathrm{O}\end{array}$ & $\begin{array}{l}11.00 \\
\mathrm{M}-\mathrm{O}\end{array}$ & $10.12 \mathrm{GH}$ & $\begin{array}{c}7.96 \\
P\end{array}$ & $\begin{array}{l}11.40 \\
\text { L-O }\end{array}$ & $\begin{array}{l}11.00 \\
\mathrm{M}-\mathrm{O}\end{array}$ & $10.12 \mathrm{GH}$ & $\begin{array}{c}7.96 \\
P\end{array}$ & $\begin{array}{c}11.40 \\
\text { L-O }\end{array}$ & $\begin{array}{l}11.00 \\
\mathrm{M}-\mathrm{O}\end{array}$ & $10.12 \mathrm{GH}$ \\
\hline N1(0.5g.) & $\begin{array}{c}10.50 \\
\text { NO }\end{array}$ & $\begin{array}{c}12.13 \\
\mathrm{I}-\mathrm{O}\end{array}$ & $\begin{array}{c}13.13 \\
\text { E-N }\end{array}$ & $11.92 \mathrm{EF}$ & $\begin{array}{l}10.83 \\
\mathrm{M}-\mathrm{O}\end{array}$ & $\begin{array}{l}10.97 \\
\mathrm{M}-\mathrm{O}\end{array}$ & $\begin{array}{c}11.57 \\
\mathrm{~L}-\mathrm{O}\end{array}$ & $11.12 \mathrm{FG}$ & $\begin{array}{c}15.33 \\
\mathrm{D}-\mathrm{F}\end{array}$ & $\begin{array}{c}15.63 \\
D-F\end{array}$ & $\begin{array}{c}18.40 \\
\text { BC }\end{array}$ & $16.46 \mathrm{C}$ \\
\hline N2(1g.) & $\begin{array}{c}10.20 \\
\text { OP }\end{array}$ & $\begin{array}{c}13.53 \\
\text { E-M }\end{array}$ & $\begin{array}{c}15.07 \\
\mathrm{D}-\mathrm{H}\end{array}$ & $12.93 \mathrm{E}$ & $\begin{array}{c}11.87 \\
\mathrm{~J}-\mathrm{O}\end{array}$ & $\begin{array}{c}12.50 \\
\mathrm{G}-\mathrm{O}\end{array}$ & $\begin{array}{l}14.37 \\
\mathrm{D}-\mathrm{K}\end{array}$ & $12.91 \mathrm{E}$ & $\begin{array}{c}14.70 \\
\text { D-I }\end{array}$ & $\begin{array}{c}15.27 \\
D-G\end{array}$ & $\begin{array}{c}14.90 \\
\text { D-H }\end{array}$ & $14.96 \mathrm{D}$ \\
\hline N3(1.5g.) & $\begin{array}{c}14.83 \\
\text { D-I }\end{array}$ & $\begin{array}{c}19.67 \\
\text { B }\end{array}$ & $\begin{array}{c}22.27 \\
\mathrm{~A}\end{array}$ & $18.92 \mathrm{~B}$ & $\begin{array}{c}12.87 \\
\text { F-O }\end{array}$ & $\begin{array}{c}14.93 \\
\mathrm{D}-\mathrm{H}\end{array}$ & $\begin{array}{c}15.87 \\
\mathrm{DE}\end{array}$ & $14.55 \mathrm{D}$ & $\begin{array}{c}12.37 \\
\mathrm{H}-\mathrm{O}\end{array}$ & $\begin{array}{c}12.90 \\
\text { F-O }\end{array}$ & $\begin{array}{c}14.53 \\
\text { D-J }\end{array}$ & $13.27 \quad \mathrm{E}$ \\
\hline N4(2g.) & $\begin{array}{c}15.47 \\
\mathrm{D}-\mathrm{F}\end{array}$ & $\begin{array}{c}22.37 \\
\mathrm{~A}\end{array}$ & $\begin{array}{c}24.37 \\
\mathrm{~A}\end{array}$ & $20.73 \mathrm{~A}$ & $\begin{array}{c}15.20 \\
D-G\end{array}$ & $\begin{array}{c}16.93 \\
\text { CD }\end{array}$ & $\begin{array}{c}19.50 \\
\text { B }\end{array}$ & $17.21 \mathrm{C}$ & $\begin{array}{c}8.06 \\
\mathrm{P}\end{array}$ & $\begin{array}{l}11.67 \\
\mathrm{~K}-\mathrm{O}\end{array}$ & $\begin{array}{c}11.77 \\
\mathrm{~K}-\mathrm{O}\end{array}$ & $\begin{array}{c}10.49 \\
\mathrm{GH}\end{array}$ \\
\hline Mean & $\begin{array}{c}11.79 \\
\mathrm{E}\end{array}$ & $\begin{array}{c}15.82 \\
\mathrm{~B} \\
\end{array}$ & $\begin{array}{c}17.17 \\
\mathrm{~A}\end{array}$ & & $\begin{array}{c}11.74 \\
\mathrm{E} \\
\end{array}$ & $\begin{array}{c}13.35 \\
\mathrm{D} \\
\end{array}$ & $\begin{array}{c}14.46 \\
\mathrm{C} \\
\end{array}$ & & $\begin{array}{c}11.69 \\
\mathrm{E}\end{array}$ & $\begin{array}{c}13.37 \\
\mathrm{D}\end{array}$ & $\begin{array}{c}14.12 \\
\text { CD } \\
\end{array}$ & \\
\hline
\end{tabular}

Means followed by a letter in common in the same column are not significantly different at 0.05 level of probability 
Table (5): Effect of nitrogen sources and rates under different levels of water field capacity on leaf fresh weight (g./seedling) of Cupressus sempervirens seedlings during the seasons of 2008-2009 and 2009-2010.

\begin{tabular}{|c|c|c|c|c|c|c|c|c|c|c|c|c|}
\hline \multicolumn{13}{|c|}{ First season } \\
\hline Fertilizer & \multicolumn{4}{|c|}{ Calcium nitrate } & \multicolumn{4}{|c|}{ Ammonium sulfate } & \multicolumn{4}{|c|}{ Ammonium nitrate } \\
\hline & $\begin{array}{l}60 \% \\
\text { F.C. }\end{array}$ & $\begin{array}{l}\text { 80\% } \\
\text { F.C. }\end{array}$ & $\begin{array}{c}100 \% \\
\text { F.C. }\end{array}$ & Mean & $\begin{array}{l}\text { 60\% } \\
\text { F.C. }\end{array}$ & $\begin{array}{l}\text { 80\% } \\
\text { F.C. }\end{array}$ & $\begin{array}{l}100 \% \\
\text { F.C. }\end{array}$ & Mean & $\begin{array}{c}60 \% \\
\text { F.C. }\end{array}$ & $\begin{array}{l}\text { 80\% } \\
\text { F.C. }\end{array}$ & $\begin{array}{c}100 \% \\
\text { F.C. }\end{array}$ & Mean \\
\hline $\begin{array}{l}\text { control } \\
\text { (zero) }\end{array}$ & $\begin{array}{c}17.67 \\
P\end{array}$ & $\begin{array}{c}19.93 \\
\text { OP }\end{array}$ & $\begin{array}{c}21.67 \\
\text { N-P }\end{array}$ & $\begin{array}{c}19.76 \\
\mathrm{H}\end{array}$ & $\begin{array}{c}17.67 \\
P\end{array}$ & $\begin{array}{c}19.93 \\
\text { OP }\end{array}$ & $\begin{array}{c}21.67 \\
\text { N-P }\end{array}$ & $19.76 \mathrm{H}$ & $\begin{array}{c}17.67 \\
P\end{array}$ & $\begin{array}{c}19.93 \\
\text { OP }\end{array}$ & $\begin{array}{c}21.67 \\
\text { N-P }\end{array}$ & $19.76 \mathrm{H}$ \\
\hline N1(0.5g.) & $\begin{array}{c}27.23 \\
\text { L-P }\end{array}$ & $\begin{array}{c}46.77 \\
\text { E-I }\end{array}$ & $\begin{array}{c}49.90 \\
C-G\end{array}$ & $\begin{array}{c}41.30 \\
\mathrm{EF}\end{array}$ & $\begin{array}{c}23.23 \\
\text { N-P }\end{array}$ & $\begin{array}{c}42.50 \\
\text { G-J }\end{array}$ & $\begin{array}{c}47.27 \\
\text { E-I }\end{array}$ & $37.67 \mathrm{~F}$ & $\begin{array}{c}30.87 \\
\text { K-N }\end{array}$ & $\begin{array}{c}48.33 \\
\text { D-H }\end{array}$ & $\begin{array}{c}56.73 \\
\text { B-D }\end{array}$ & 45.31DE \\
\hline N2(1g.) & $\begin{array}{c}38.50 \\
\mathrm{I}-\mathrm{K}\end{array}$ & $\begin{array}{c}55.30 \\
B-E\end{array}$ & $\begin{array}{c}61.23 \\
\text { B }\end{array}$ & $51.68 \mathrm{C}$ & $\begin{array}{c}34.83 \\
\mathrm{~J}-\mathrm{M}\end{array}$ & $\begin{array}{c}52.27 \\
\text { B-F }\end{array}$ & $\begin{array}{c}59.57 \\
\text { B }\end{array}$ & 48.89CD & $\begin{array}{c}27.70 \\
\mathrm{~L}-\mathrm{O}\end{array}$ & $\begin{array}{c}39.17 \\
\text { H-K }\end{array}$ & $\begin{array}{c}46.30 \\
\text { E-I }\end{array}$ & $37.72 \mathrm{~F}$ \\
\hline N3(1.5g.) & $\begin{array}{c}49.57 \\
C-G\end{array}$ & $\begin{array}{c}61.47 \\
\text { B }\end{array}$ & $\begin{array}{c}75.27 \\
\text { A }\end{array}$ & $\begin{array}{c}62.10 \\
\text { B }\end{array}$ & $\begin{array}{c}44.47 \\
\text { F-I }\end{array}$ & $\begin{array}{l}57.60 \\
\text { B-D }\end{array}$ & $\begin{array}{c}74.57 \\
\text { A }\end{array}$ & $58.88 \mathrm{~B}$ & $\begin{array}{c}22.03 \\
\text { N-P }\end{array}$ & $\begin{array}{c}25.87 \\
\text { M-P }\end{array}$ & $\begin{array}{c}35.30 \\
\text { J-L }\end{array}$ & $27.73 \mathrm{G}$ \\
\hline N4(2g.) & $\begin{array}{c}58.87 \\
\text { BC }\end{array}$ & $\begin{array}{c}72.60 \\
\text { A }\end{array}$ & $\begin{array}{c}79.30 \\
\text { A }\end{array}$ & $\begin{array}{c}70.26 \\
\text { A }\end{array}$ & $\begin{array}{c}55.73 \\
\text { B-E }\end{array}$ & $\begin{array}{c}71.23 \\
\mathrm{~A}\end{array}$ & $\begin{array}{c}78.37 \\
\text { A }\end{array}$ & $58.88 \mathrm{~B}$ & $\begin{array}{c}15.26 \\
P Q\end{array}$ & $\begin{array}{c}22.00 \\
\text { N-P }\end{array}$ & $\begin{array}{c}28.23 \\
\text { L-O }\end{array}$ & $21.83 \mathrm{H}$ \\
\hline Mean & $\begin{array}{r}38.37 \\
\mathrm{C}\end{array}$ & $\begin{array}{r}51.21 \\
\text { B }\end{array}$ & $\begin{array}{r}57.47 \\
\mathrm{~A}\end{array}$ & & $\begin{array}{c}35.19 \\
\mathrm{C}\end{array}$ & $\begin{array}{r}48.71 \\
\text { B }\end{array}$ & $\begin{array}{r}56.33 \\
\mathrm{~A}\end{array}$ & & $\begin{array}{c}22.71 \\
\mathrm{E}\end{array}$ & $\begin{array}{c}31.06 \\
\text { D }\end{array}$ & $\begin{array}{r}37.69 \\
\mathrm{C}\end{array}$ & \\
\hline \multicolumn{13}{|c|}{ Second season } \\
\hline Fertilizer & \multicolumn{4}{|c|}{ Calcium nitrate } & \multicolumn{4}{|c|}{ Ammonium sulfate } & \multicolumn{4}{|c|}{ Ammonium nitrate } \\
\hline & $\begin{array}{l}60 \% \\
\text { F.C. }\end{array}$ & $\begin{array}{l}80 \% \\
\text { F.C. }\end{array}$ & $\begin{array}{c}100 \% \\
\text { F.C. }\end{array}$ & Mean & $\begin{array}{l}60 \% \\
\text { F.C. }\end{array}$ & $\begin{array}{l}\text { 80\% } \\
\text { F.C. }\end{array}$ & $\begin{array}{l}100 \% \\
\text { F.C. }\end{array}$ & Mean & $\begin{array}{c}60 \% \\
\text { F.C. }\end{array}$ & $\begin{array}{l}80 \% \\
\text { F.C. }\end{array}$ & $\begin{array}{c}100 \% \\
\text { F.C. }\end{array}$ & Mean \\
\hline $\begin{array}{l}\text { control } \\
\text { (zero) }\end{array}$ & $\begin{array}{c}18.43 \\
\mathrm{O}\end{array}$ & $\begin{array}{c}24.47 \\
\mathrm{~L}-\mathrm{O}\end{array}$ & $\begin{array}{c}26.23 \\
\text { K-O }\end{array}$ & $\begin{array}{c}23.04 \\
\text { I }\end{array}$ & $\begin{array}{c}18.43 \\
\mathrm{O}\end{array}$ & $\begin{array}{c}24.47 \\
\text { L-O }\end{array}$ & $\begin{array}{c}26.23 \\
\text { K-O }\end{array}$ & $\begin{array}{c}23.04 \\
\text { I }\end{array}$ & $\begin{array}{c}18.43 \\
\mathrm{O}\end{array}$ & $\begin{array}{c}24.47 \\
\mathrm{~L}-\mathrm{O}\end{array}$ & $\begin{array}{c}26.23 \\
\mathrm{~K}-\mathrm{O}\end{array}$ & $\begin{array}{c}23.04 \\
\text { I }\end{array}$ \\
\hline N1(0.5g.) & $\begin{array}{c}31.83 \\
\mathrm{I}-\mathrm{M}\end{array}$ & $\begin{array}{c}51.90 \\
\text { E-G }\end{array}$ & $\begin{array}{c}55.93 \\
C-G\end{array}$ & $\begin{array}{c}46.56 \\
\text { EF }\end{array}$ & $\begin{array}{c}27.57 \\
\mathrm{~K}-\mathrm{O}\end{array}$ & $\begin{array}{c}48.27 \\
\mathrm{GH}\end{array}$ & $\begin{array}{c}53.70 \\
D-G\end{array}$ & $\begin{array}{c}43.18 \\
\text { FG }\end{array}$ & $\begin{array}{c}34.03 \\
\text { I-L }\end{array}$ & $\begin{array}{c}49.20 \\
\text { F-H }\end{array}$ & $\begin{array}{c}58.50 \\
B-G\end{array}$ & $\begin{array}{c}47.24 \\
\mathrm{EF}\end{array}$ \\
\hline N2(1g.) & $\begin{array}{c}35.57 \\
\mathrm{I}-\mathrm{K}\end{array}$ & $\begin{array}{c}60.50 \\
\text { B-E }\end{array}$ & $\begin{array}{l}63.73 \\
\text { B-D }\end{array}$ & $\begin{array}{c}53.27 \\
\text { D }\end{array}$ & $\begin{array}{c}29.50 \\
\mathrm{~J}-\mathrm{N}\end{array}$ & $\begin{array}{c}57.37 \\
\text { B-G }\end{array}$ & $\begin{array}{c}61.53 \\
\text { B-E }\end{array}$ & $\begin{array}{c}49.47 \\
\mathrm{DE}\end{array}$ & $\begin{array}{c}29.13 \\
\text { J-N }\end{array}$ & $\begin{array}{c}41.07 \\
\text { HI }\end{array}$ & $\begin{array}{c}51.67 \\
\text { E-G }\end{array}$ & $\begin{array}{c}40.62 \\
\mathrm{G}\end{array}$ \\
\hline N3(1.5g.) & $\begin{array}{c}57.93 \\
\text { B-G }\end{array}$ & $\begin{array}{l}64.27 \\
\text { BCD }\end{array}$ & $\begin{array}{l}67.40 \\
\text { B-D }\end{array}$ & $\begin{array}{c}63.20 \\
\text { BC }\end{array}$ & $\begin{array}{c}53.77 \\
D-G\end{array}$ & $\begin{array}{c}61.40 \\
\text { B-E }\end{array}$ & $\begin{array}{c}65.20 \\
\mathrm{BC}\end{array}$ & $\begin{array}{c}60.12 \\
C\end{array}$ & $\begin{array}{c}20.43 \\
\text { NO }\end{array}$ & $\begin{array}{c}30.53 \\
\mathrm{~J}-\mathrm{N}\end{array}$ & $\begin{array}{c}38.00 \\
\text { IJ }\end{array}$ & $\begin{array}{c}29.66 \\
H\end{array}$ \\
\hline N4(2g.) & $\begin{array}{c}59.73 \\
\text { B-F }\end{array}$ & $\begin{array}{c}65.57 \\
\text { BC }\end{array}$ & $\begin{array}{c}84.57 \\
\text { A }\end{array}$ & $\begin{array}{c}69.96 \\
\text { A }\end{array}$ & $\begin{array}{c}55.47 \\
\text { C-G }\end{array}$ & $\begin{array}{c}62.37 \\
\text { B-E }\end{array}$ & $\begin{array}{c}80.33 \\
\text { A }\end{array}$ & $\begin{array}{c}66.06 \\
\mathrm{AB}\end{array}$ & $\begin{array}{c}16.23 \\
\mathrm{O}\end{array}$ & $\begin{array}{l}22.73 \\
\text { M-O }\end{array}$ & $\begin{array}{c}28.00 \\
\text { J-O }\end{array}$ & $\begin{array}{c}21.83 \\
\text { I }\end{array}$ \\
\hline Mean & $\begin{array}{c}40.70 \\
\mathrm{C}\end{array}$ & $\begin{array}{c}53.34 \\
\text { B }\end{array}$ & $\begin{array}{c}59.57 \\
\text { A }\end{array}$ & & $\begin{array}{c}36.95 \\
\text { CD }\end{array}$ & $\begin{array}{c}50.74 \\
\text { B }\end{array}$ & $\begin{array}{c}57.40 \\
\text { A }\end{array}$ & & $\begin{array}{c}23.67 \\
E\end{array}$ & $\begin{array}{c}33.60 \\
\text { D }\end{array}$ & $\begin{array}{c}40.48 \\
\text { C }\end{array}$ & \\
\hline
\end{tabular}

Means followed by a letter in common in the same column are not significantly different at 0.05 level of probability 
Table (6): Effect of nitrogen sources and rates under different levels of water field capacity on leaf dry weight (g./seedling) of Cupressus sempervirens seedlings during the seasons of 2008-2009 and 2009-2010.

\begin{tabular}{|c|c|c|c|c|c|c|c|c|c|c|c|c|}
\hline \multicolumn{13}{|c|}{ First season } \\
\hline Fertilizer & \multicolumn{4}{|c|}{ Calcium nitrate } & \multicolumn{4}{|c|}{ Ammonium sulfate } & \multicolumn{4}{|c|}{ Ammonium nitrate } \\
\hline & $\begin{array}{l}60 \% \\
\text { F.C. }\end{array}$ & $\begin{array}{c}80 \% \\
\text { F.C. }\end{array}$ & $\begin{array}{c}100 \% \\
\text { F.C. }\end{array}$ & Mean & $\begin{array}{l}60 \% \\
\text { F.C. }\end{array}$ & $\begin{array}{l}\text { 80\% } \\
\text { F.C. }\end{array}$ & $\begin{array}{l}100 \% \\
\text { F.C. }\end{array}$ & Mean & $\begin{array}{l}60 \% \\
\text { F.C. }\end{array}$ & $\begin{array}{l}80 \% \\
\text { F.C. }\end{array}$ & $\begin{array}{c}100 \% \\
\text { F.C. }\end{array}$ & Mean \\
\hline $\begin{array}{l}\text { control } \\
\text { (zero) }\end{array}$ & $\begin{array}{c}8.93 \\
\mathrm{~L}\end{array}$ & $\begin{array}{c}11.40 \\
\mathrm{KL}\end{array}$ & $\begin{array}{c}11.50 \\
\text { KL }\end{array}$ & $10.61 \mathrm{G}$ & $\begin{array}{c}8.93 \\
\mathrm{~L}\end{array}$ & $\begin{array}{c}11.40 \\
\mathrm{KL}\end{array}$ & $\begin{array}{c}11.50 \\
\mathrm{KL}\end{array}$ & $10.61 \mathrm{G}$ & $\begin{array}{c}8.93 \\
\mathrm{~L}\end{array}$ & $\begin{array}{c}11.40 \\
\mathrm{KL}\end{array}$ & $\begin{array}{c}11.50 \\
\text { KL }\end{array}$ & $10.61 \mathrm{G}$ \\
\hline N1(0.5g.) & $\begin{array}{c}11.87 \\
\mathrm{~J}-\mathrm{L}\end{array}$ & $\begin{array}{c}22.20 \\
\text { E-H }\end{array}$ & $\begin{array}{c}24.00 \\
D-G\end{array}$ & $19.36 \mathrm{E}$ & $\begin{array}{c}12.20 \\
\mathrm{~J}-\mathrm{L}\end{array}$ & $\begin{array}{c}22.23 \\
\text { E-H }\end{array}$ & $\begin{array}{c}23.17 \\
\text { E-H }\end{array}$ & $19.20 \mathrm{E}$ & $\begin{array}{c}14.27 \\
\text { JK }\end{array}$ & $\begin{array}{c}24.00 \\
D-G\end{array}$ & $\begin{array}{c}26.00 \\
C-E\end{array}$ & 21.42DE \\
\hline N2(1g.) & $\begin{array}{c}21.67 \\
\text { E-H }\end{array}$ & $\begin{array}{c}24.33 \\
\mathrm{D}-\mathrm{F} \\
\end{array}$ & $\begin{array}{c}30.20 \\
\mathrm{C} \\
\end{array}$ & $25.33 \mathrm{CD}$ & $\begin{array}{c}18.87 \\
\text { HI }\end{array}$ & $\begin{array}{c}25.63 \\
\text { C-E }\end{array}$ & $\begin{array}{c}28.43 \\
\text { CD }\end{array}$ & $24.31 \mathrm{C}$ & $\begin{array}{c}13.70 \\
\mathrm{~J}-\mathrm{L}\end{array}$ & $\begin{array}{c}19.93 \\
\text { F-I }\end{array}$ & $\begin{array}{c}24.13 \\
\mathrm{D}-\mathrm{G}\end{array}$ & $19.26 \mathrm{E}$ \\
\hline N3(1.5g.) & $\begin{array}{c}22.97 \\
\text { E-H }\end{array}$ & $\begin{array}{c}29.53 \\
C\end{array}$ & $\begin{array}{c}35.53 \\
\mathrm{AB}\end{array}$ & 29.34B & $\begin{array}{c}23.80 \\
D-G\end{array}$ & $\begin{array}{c}28.73 \\
\text { CD }\end{array}$ & $\begin{array}{c}37.73 \\
\text { AB }\end{array}$ & $30.09 \mathrm{~B}$ & $\begin{array}{c}10.83 \\
\text { KL }\end{array}$ & $\begin{array}{c}12.63 \\
\mathrm{~J}-\mathrm{L}\end{array}$ & $\begin{array}{c}19.13 \\
\text { G-I }\end{array}$ & $14.20 \mathrm{~F}$ \\
\hline N4(2g.) & $\begin{array}{c}28.47 \\
\text { CD }\end{array}$ & $\begin{array}{c}34.53 \\
\text { B }\end{array}$ & $\begin{array}{c}40.20 \\
\mathrm{~A}\end{array}$ & $34.40 \mathrm{~A}$ & $\begin{array}{c}26.63 \\
\text { C-E }\end{array}$ & $\begin{array}{c}36.53 \\
\mathrm{AB}\end{array}$ & $\begin{array}{c}38.93 \\
\mathrm{AB}\end{array}$ & $34.03 \mathrm{~A}$ & $\begin{array}{c}6.96 \\
\mathrm{M}\end{array}$ & $\begin{array}{c}11.10 \\
\mathrm{KL}\end{array}$ & $\begin{array}{c}12.57 \\
\mathrm{~J}-\mathrm{L}\end{array}$ & $10.20 \mathrm{G}$ \\
\hline Mean & $\begin{array}{c}18.78 \\
\mathrm{C} \\
\end{array}$ & $\begin{array}{c}24.40 \\
\text { B } \\
\end{array}$ & $\begin{array}{c}28.29 \\
\mathrm{~A} \\
\end{array}$ & & $\begin{array}{c}18.09 \\
\mathrm{C} \\
\end{array}$ & $\begin{array}{r}24.91 \\
\text { B }\end{array}$ & $\begin{array}{c}27.95 \\
\mathrm{~A} \\
\end{array}$ & & $\begin{array}{c}10.94 \\
\mathrm{E} \\
\end{array}$ & $\begin{array}{c}15.81 \\
\mathrm{D} \\
\end{array}$ & $\begin{array}{c}18.67 \\
\mathrm{C} \\
\end{array}$ & \\
\hline \multicolumn{13}{|c|}{ Second season } \\
\hline Fertilizer & \multicolumn{4}{|c|}{ Calcium nitrate } & \multicolumn{4}{|c|}{ Ammonium sulfate } & \multicolumn{4}{|c|}{ Ammonium nitrate } \\
\hline & $\begin{array}{c}60 \% \\
\text { F.C. }\end{array}$ & $\begin{array}{l}80 \% \\
\text { F.C. }\end{array}$ & $\begin{array}{c}100 \% \\
\text { F.C. }\end{array}$ & Mean & $\begin{array}{l}60 \% \\
\text { F.C. }\end{array}$ & $\begin{array}{l}80 \% \\
\text { F.C. }\end{array}$ & $\begin{array}{l}100 \% \\
\text { F.C. }\end{array}$ & Mean & $\begin{array}{c}60 \% \\
\text { F.C. }\end{array}$ & $\begin{array}{l}80 \% \\
\text { F.C. }\end{array}$ & $\begin{array}{c}100 \% \\
\text { F.C. }\end{array}$ & Mean \\
\hline $\begin{array}{l}\text { control } \\
\text { (zero) }\end{array}$ & $\begin{array}{c}11.13 \\
\mathrm{H}\end{array}$ & $\begin{array}{c}12.53 \\
\mathrm{GH}\end{array}$ & $\begin{array}{c}12.77 \\
\mathrm{GH}\end{array}$ & $12.14 \mathrm{EF}$ & $\begin{array}{c}11.13 \\
\mathrm{H}\end{array}$ & $\begin{array}{c}12.53 \\
\mathrm{GH}\end{array}$ & $\begin{array}{c}12.77 \\
\mathrm{GH}\end{array}$ & $12.14 \mathrm{EF}$ & $\begin{array}{c}11.13 \\
\mathrm{H}\end{array}$ & $\begin{array}{c}12.53 \\
\mathrm{GH}\end{array}$ & $\begin{array}{c}12.77 \\
\mathrm{GH}\end{array}$ & $12.14 \mathrm{EF}$ \\
\hline N1(0.5g.) & $\begin{array}{c}15.17 \\
\text { F-H }\end{array}$ & $\begin{array}{c}23.80 \\
\text { DE }\end{array}$ & $\begin{array}{c}26.70 \\
B-E\end{array}$ & 21.89CD & $\begin{array}{c}11.23 \\
\mathrm{GH}\end{array}$ & $\begin{array}{c}23.47 \\
\mathrm{E}\end{array}$ & $\begin{array}{c}25.43 \\
\text { C-E }\end{array}$ & $20.04 \mathrm{D}$ & $\begin{array}{c}17.07 \\
\text { FG }\end{array}$ & $\begin{array}{c}26.70 \\
B-E\end{array}$ & $\begin{array}{c}29.37 \\
\text { B-D }\end{array}$ & $24.38 \mathrm{C}$ \\
\hline N2(1g.) & $\begin{array}{c}15.20 \\
\text { F-H }\end{array}$ & $\begin{array}{c}28.07 \\
\text { B-E }\end{array}$ & $\begin{array}{c}28.27 \\
\text { B-E }\end{array}$ & $23.84 \mathrm{C}$ & $\begin{array}{c}14.80 \\
\text { F-H }\end{array}$ & $\begin{array}{c}27.30 \\
B-E\end{array}$ & $\begin{array}{c}27.97 \\
B-E\end{array}$ & $23.36 \mathrm{C}$ & $\begin{array}{c}15.37 \\
\text { F-H }\end{array}$ & $\begin{array}{c}18.63 \\
F\end{array}$ & $\begin{array}{c}25.63 \\
C-E\end{array}$ & $19.88 \mathrm{D}$ \\
\hline N3(1.5g.) & $\begin{array}{c}26.73 \\
\text { B-E }\end{array}$ & $\begin{array}{c}29.10 \\
\text { B-E }\end{array}$ & $\begin{array}{c}27.50 \\
\text { B-E }\end{array}$ & 27.78 B & $\begin{array}{c}27.57 \\
\text { B-E }\end{array}$ & $\begin{array}{c}31.83 \\
\mathrm{~B} \\
\end{array}$ & $\begin{array}{c}30.07 \\
\mathrm{BC}\end{array}$ & $29.82 \mathrm{AB}$ & $\begin{array}{c}10.47 \\
\mathrm{H}\end{array}$ & $\begin{array}{c}14.83 \\
\text { F-H }\end{array}$ & $\begin{array}{c}17.00 \\
\text { FG }\end{array}$ & $14.10 \mathrm{E}$ \\
\hline N4(2g.) & $\begin{array}{c}25.03 \\
\text { C-E }\end{array}$ & $\begin{array}{c}25.63 \\
\text { C-E }\end{array}$ & $\begin{array}{c}39.30 \\
\mathrm{~A}\end{array}$ & 29.99AB & $\begin{array}{c}27.23 \\
\text { B-E }\end{array}$ & $\begin{array}{c}30.77 \\
\mathrm{BC}\end{array}$ & $\begin{array}{c}39.47 \\
\mathrm{~A}\end{array}$ & $32.49 \mathrm{~A}$ & $\begin{array}{c}7.46 \\
\text { I }\end{array}$ & $\begin{array}{c}11.30 \\
\mathrm{GH}\end{array}$ & $\begin{array}{c}13.87 \\
\text { F-H }\end{array}$ & $\begin{array}{c}10.87 \\
\mathrm{~F} \\
\end{array}$ \\
\hline Mean & $\begin{array}{c}18.65 \\
\text { CD } \\
\end{array}$ & $\begin{array}{c}23.83 \\
\mathrm{~B} \\
\end{array}$ & $\begin{array}{r}26.91 \\
\mathrm{~A} \\
\end{array}$ & & $\begin{array}{c}18.39 \\
\text { CD }\end{array}$ & $\begin{array}{c}25.18 \\
\text { AB }\end{array}$ & $\begin{array}{c}27.14 \\
\mathrm{~A} \\
\end{array}$ & & $\begin{array}{c}12.30 \\
\mathrm{E} \\
\end{array}$ & $\begin{array}{c}16.80 \\
\mathrm{D} \\
\end{array}$ & $\begin{array}{c}19.73 \\
\mathrm{C} \\
\end{array}$ & \\
\hline
\end{tabular}

Means followed by a letter in common in the same column are not significantly different at 0.05 level of probability 
Table (7): Effect of nitrogen sources under different levels of field capaciety on nitrogen, phosphorus, potassium content (\%) and porline (mg./g. D.W.) In the leaves of Cupressus sempervirens seedlings during the seasons of 2008-2009 and 2009-2010.

\begin{tabular}{|c|c|c|c|c|c|c|c|c|c|c|c|c|c|}
\hline \multicolumn{14}{|c|}{ First season } \\
\hline \multirow{2}{*}{\multicolumn{2}{|c|}{ Treatments }} & \multicolumn{3}{|c|}{ Nitrogen \% } & \multicolumn{3}{|c|}{ Phosphorus\% } & \multicolumn{3}{|c|}{ Potassium \% } & \multicolumn{3}{|c|}{ Proline (mg./g. D.W.) } \\
\hline & & $\begin{array}{l}60 \% \\
\text { F.C. }\end{array}$ & $\begin{array}{c}80 \% \\
\text { F.C. }\end{array}$ & $\begin{array}{r}100 \% \\
\text { F.C. }\end{array}$ & $\begin{array}{l}60 \% \\
\text { F.C. }\end{array}$ & $\begin{array}{l}80 \% \\
\text { F.C. }\end{array}$ & $\begin{array}{r}100 \% \\
\text { F.C. }\end{array}$ & $\begin{array}{l}60 \% \\
\text { F.C. }\end{array}$ & $\begin{array}{c}80 \% \\
\text { F.C. }\end{array}$ & $\begin{array}{r}100 \% \\
\text { F.C. }\end{array}$ & $\begin{array}{l}60 \% \\
\text { F.C. }\end{array}$ & $\begin{array}{l}80 \% \\
\text { F.C. }\end{array}$ & $\begin{array}{r}100 \% \\
\text { F.C. }\end{array}$ \\
\hline \multicolumn{2}{|c|}{ Control (zero) } & 0.69 & 0.70 & 0.70 & 0.20 & 0.20 & 0.21 & 0.32 & 0.32 & 0.33 & 0.26 & 0.23 & 0.22 \\
\hline \multirow{4}{*}{$\begin{array}{l}\text { Calcium } \\
\text { nitrate }\end{array}$} & N1(0.5g.) & 1.09 & 1.09 & 1.12 & 0.21 & 0.21 & 0.22 & 0.30 & 0.34 & 0.34 & 0.20 & 0.14 & 0.10 \\
\hline & N2(1g.) & 1.30 & 1.33 & 1.33 & 0.23 & 0.26 & 0.26 & 0.33 & 0.32 & 0.37 & 0.21 & 0.15 & 0.10 \\
\hline & N3(1.5g.) & 1.40 & 1.42 & 1.42 & 0.30 & 0.30 & 0.36 & 0.37 & 0.44 & 0.45 & 0.23 & 0.15 & 0.13 \\
\hline & N4(2g.) & 1.97 & 1.97 & 2.01 & 0.34 & 0.35 & 0.35 & 0.31 & 0.32 & 0.36 & 0.23 & 0.15 & 0.15 \\
\hline \multirow{4}{*}{$\begin{array}{l}\text { Ammonium } \\
\text { sulfate }\end{array}$} & N1(0.5g.) & 0.99 & 0.99 & 0.99 & 0.20 & 0.20 & 0.20 & 0.57 & 0.59 & 0.59 & 0.27 & 0.24 & 0.25 \\
\hline & N2(1g.) & 1.05 & 1.09 & 1.10 & 0.21 & 0.24 & 0.27 & 0.45 & 0.47 & 0.50 & 0.26 & 0.26 & 0.25 \\
\hline & N3(1.5g.) & 1.09 & 1.12 & 1.14 & 0.22 & 0.24 & 0.28 & 0.40 & 0.42 & 0.48 & 0.33 & 0.27 & 0.26 \\
\hline & N4(2g.) & 1.42 & 1.43 & 1.60 & 0.22 & 0.28 & 0.28 & 0.36 & 0.40 & 0.46 & 0.36 & 0.29 & 0.29 \\
\hline \multirow{4}{*}{$\begin{array}{l}\text { Ammonium } \\
\text { nitrate }\end{array}$} & N1(0.5g.) & 0.98 & 0.98 & 1.01 & 0.20 & 0.20 & 0.20 & 0.50 & 0.50 & 0.50 & 0.24 & 0.24 & 0.25 \\
\hline & N2(1g.) & 1.04 & 1.10 & 1.10 & 0.20 & 0.26 & 0.26 & 0.43 & 0.42 & 0.52 & 0.29 & 0.24 & 0.25 \\
\hline & N3(1.5g.) & 1.01 & 1.01 & 1.01 & 0.20 & 0.21 & 0.21 & 0.37 & 0.39 & 0.39 & 0.39 & 0.28 & 0.26 \\
\hline & N4(2g.) & 0.88 & 0.88 & 0.99 & 0.20 & 0.20 & 0.20 & 0.31 & 0.32 & 0.33 & 0.40 & 0.30 & 0.28 \\
\hline
\end{tabular}

\begin{tabular}{|c|c|c|c|c|c|c|c|c|c|c|c|c|c|}
\hline \multirow{3}{*}{\multicolumn{2}{|c|}{ Treatments }} & \multicolumn{9}{|c|}{ Second season } & \multirow{2}{*}{\multicolumn{3}{|c|}{ Proline (mg./g. D.W.) }} \\
\hline & & \multicolumn{3}{|c|}{ Nitrogen \% } & \multicolumn{3}{|c|}{ Phosphorus\% } & \multicolumn{3}{|c|}{ Potassium \% } & & & \\
\hline & & $\begin{array}{l}\text { 60\% } \\
\text { F.C. }\end{array}$ & $\begin{array}{l}80 \% \\
\text { F.C. }\end{array}$ & $\begin{array}{l}100 \% \\
\text { F.C. }\end{array}$ & $\begin{array}{l}60 \% \\
\text { F.C. }\end{array}$ & $\begin{array}{l}80 \% \\
\text { F.C. }\end{array}$ & $\begin{array}{c}100 \% \\
\text { F.C. }\end{array}$ & $\begin{array}{l}60 \% \\
\text { F.C. }\end{array}$ & $\begin{array}{c}\text { 80\% } \\
\text { F.C. }\end{array}$ & $\begin{array}{c}100 \% \\
\text { F.C. }\end{array}$ & $\begin{array}{l}\text { 60\% } \\
\text { F.C. }\end{array}$ & $\begin{array}{c}80 \% \\
\text { F.C. }\end{array}$ & $\begin{array}{r}100 \% \\
\text { F.C. }\end{array}$ \\
\hline \multicolumn{2}{|c|}{ Control (zero) } & 0.59 & 0.61 & 0.60 & 0.20 & 0.20 & 0.20 & 0.30 & 0.30 & 0.30 & 0.26 & 0.20 & 0.20 \\
\hline \multirow{4}{*}{$\begin{array}{l}\text { Calcium } \\
\text { nitrate }\end{array}$} & N1(0.5g.) & 1.09 & 1.16 & 1.50 & 0.21 & 0.23 & 0.23 & 0.30 & 0.30 & 0.34 & 0.20 & 0.14 & 0.12 \\
\hline & N2(1g.) & 1.13 & 1.49 & 1.99 & 0.23 & 0.25 & 0.28 & 0.32 & 0.32 & 0.34 & 0.20 & 0.14 & 0.12 \\
\hline & N3(1.5g.) & 1.39 & 1.63 & 2.07 & 0.31 & 0.32 & 0.34 & 0.46 & 0.38 & 0.39 & 0.20 & 0.16 & 0.12 \\
\hline & N4(2g.) & 1.69 & 2.00 & 2.71 & 0.31 & 0.33 & 0.34 & 0.37 & 0.45 & 0.49 & 0.20 & 0.16 & 0.13 \\
\hline \multirow{4}{*}{$\begin{array}{l}\text { Ammonium } \\
\text { sulfate }\end{array}$} & N1(0.5g.) & 1.01 & 1.01 & 1.02 & 0.20 & 0.21 & 0.21 & 0.51 & 0.55 & 0.65 & 0.26 & 0.23 & 0.24 \\
\hline & N2(1g.) & 1.05 & 1.14 & 1.14 & 0.21 & 0.22 & 0.26 & 0.48 & 0.59 & 0.59 & 0.26 & 024 & 0.24 \\
\hline & N3(1.5g.) & 1.17 & 1.20 & 1.18 & 0.21 & 0.22 & 0.26 & 0.37 & 0.48 & 0.49 & 0.27 & 0.27 & 0.27 \\
\hline & N4(2g.) & 1.66 & 1.97 & 1.52 & 0.21 & 0.23 & 0.26 & 0.32 & 0.48 & 0.49 & 0.29 & 0.24 & 0.27 \\
\hline \multirow{4}{*}{$\begin{array}{l}\text { Ammonium } \\
\text { nitrate }\end{array}$} & N1(0.5g.) & 0.98 & 1.00 & 1.00 & 0.20 & 0.20 & 0.20 & 0.36 & 0.57 & 0.69 & 0.26 & 0.25 & 0.20 \\
\hline & N2(1g.) & 1.02 & 1.10 & 0.99 & 0.20 & 0.23 & 0.23 & 0.30 & 0.40 & 0.60 & 0.29 & 0.25 & 0.22 \\
\hline & N3(1.5g.) & 1.01 & 1.01 & 0.98 & 0.20 & 0.20 & 0.21 & 0.30 & 0.40 & 0.40 & 0.40 & 0.28 & 0.27 \\
\hline & N4(2g.) & 0.78 & 0.83 & 0.90 & 0.20 & 0.20 & 0.20 & 0.31 & 0.32 & 0.41 & 0.42 & 0.30 & 0.29 \\
\hline
\end{tabular}


$\mathrm{N} /$ seedling with $100 \%$ field capacity level in the first and the second seasons, respectively.

The aforementioned results agreed with many investigations which indicated that low level of water field capacity decreased vegetative growth. Wang et al. (2004) measured photosynthesis on five pine species. Their results showed that the net photosynthetic rate was significantly reduced $34.43 \%$ under water stress. In this respect, De Diego et al. (2012) studied the changes of leaf water potential, hydraulic conductance $\left(K_{\text {leaf }}\right)$, stomatal conductance and phytohormones under drought in Pinus radiate. They found that drought decreased cytokinin levels in the needles parallel to $K_{\text {leaf }}$ and gas exchange. Also, Yang et al. (2012) investigated the ecophysiological responses of Abies fabri seedlings to drought, nitrogen addition alone, and the combination of these treatments, and their results showed that the applied nitrogen improved plant water use efficiency and $\mathrm{N}$ accumulation in plant organs under drought conditions. Especially under drought conditions, more $\mathrm{N}$ was concentrated into needles by applied nitrogen as compared with other organs.

Calcium ammonium nitrate was the best nitrogen resource to improve plant growth, Sannappa et al. (2000) determined the efficiency of different sources of nitrogen fertilizers on the yield and quality of mulberry, such as calcium ammonium nitrate (CAN), ammonium chloride and ammonium sulfate. They observed that leaf yield had maximum values under the application of CAN. Krause et al. (2012) investigated Picea abies responses to N addition, and found that foliar analyses showed an increase in dry mass tree height and leaf area index.

\subsection{Chemical composition \\ 3.2.1. Nitrogen content}

Data presented in Table (7) showed that, an increase in leaf $\mathrm{N}$ content with the increase of calcium nitrate and ammonium sulfate fertilizer rate, but in the case of ammonium nitrate, the increase remained to the rate of 1.5 and the decrease to the rate of $2.0 \mathrm{~g} \mathrm{~N} /$ seedling in the first and the second seasons, respectively.

\subsubsection{Phosphorus content}

Data in Table (7) illustrated that, calcium nitrate treatment slightly increased leaf $\mathrm{P}$ content in comparison with the two other nitrogen sources under different levels of field capacity in both seasons .

\subsection{Potassium content}

According to the data presented in Table (7), all fertilizer treatments increased potassium content in leaves as compared to the control which gave the lowest values under different levels of water field capacity in the two seasons.

\subsection{Proline content}

Data presented in Table (7), showed that, treatment with ammonium nitrate and ammonium sulfate increased proline content in the leaves in all fertilizer rates under all field capacity levels as compared to the control, whereas, calcium nitrate decreased it.

For the interaction between $\mathrm{N}$ fertilizer rates and water field capacity levels, the highest value of proline was found at the rate of $2.0 \mathrm{~g} \mathrm{~N} / \mathrm{seedling}$ of ammonium nitrate under $60 \%$ of field capacity level $(0.40$ and $0.42 \mathrm{mg} / \mathrm{gm} \mathrm{dw}$.$) in the first and the$ second seasons, respectively. On the other side, the lowest value was obtained at the rate of $0.5 \mathrm{~g}$ $\mathrm{N} /$ seedling of calcium nitrate under $100 \%$ field capacity level.

In general, proline content in the leaves showed the highest values under $60 \%$ field capacity in comparison with the two other levels in all fertilizer rates in different nitrogen sources.

In accordance with these results Shvaleva et al. (2005) observed that, there were increases in concentrations of proline in two Eucalyptus globulus leaves. Sannappa et al. (2000) determined the efficiency of different sources of nitrogen fertilizers on the mulberry. The foliar constituents of mulberry leaf (total carbohydrates, proline, N, P, K, $\mathrm{Ca}, \mathrm{Mg}$ and $\mathrm{S}$ ), maximum concentrations were recorded with calcium ammonium nitrate application.

As a recommendation, in abundant water or water deficit conditions, application of calcium nitrate is favorable for Cupressus sempervirens seedlings growth as compared with application of ammonium sulfate and ammonium nitrate.

\section{REFERENCES}

Andivia E., Márquez-García B., Vázquez-Piqué J., Córdoba F. and Fernández M. (2012). Autumn fertilization with nitrogen improves nutritional status, cold hardiness and the oxidative stress response of Holm oak (Quercus ilex ssp. ballota [Desf.] Samp) nursery seedlings. Trees, 26 (2): 311-320. 
Aromatherapy Global Online Research Archive (2000). www.agoraindex.org/cypress.ht.

Bacelar E. L. V. A., Moutinho-Pereira J. M., Gonçalves, B. M. C. , Brito, C.V. Q., GomesLaranjo, J., Ferreira, H. M. F. and Carlos M. C. (2012). Water Use Strategies of Plants Under Drought Conditions. Plant Responses to Drought Stress, pp 145- 170.

Bates L. S., Waldren R. P. and Teare L. D. (1973). Rapid determination of free proline for water stress studies. Plant and Soil, 39: 205-207.

Black C. A. (1965). Methods of Soil Analysis part1 Physical and Mineralogical Properties Including a Statistics of Measurement and Sampling. Amer. Soc. Agron. Inc. Pub. Wisconsin. U.S.A.

Brown J. D. and Lilliland, O. (1946). Rapid determination of potassium and sodium in plant material and soil extracts by Flamephotometry. Proc. Amer. Soc. Hort. Sci., 48: 341-346.

Coder K. D. (1999). Drought Damage to Trees. www.forestry.uga.edu.

De-Diego N., Pérez-Alfocea F., Cantero E., Lacuesta M. and Moncaleán P. (2012). Physiological response to drought in radiata pine: phytohormone implication at leaf level. Tree Physiology, 32(4): 435-449.

Eldhuset T. D., Nagy N. E., Volařík D., Børja I., Gebauer R., Yakovlev, I. A. and Krokene, P. (2013). Drought affects tracheid structure, dehydrin expression, and above- and belowground growth in 5-year-old Norway spruce. Plant and Soil, 366 (1-2): 305-320.

Follett R. H. (1981). Fertilizers and soil amendments. Prentice- Hall, Inc., Englewood Cliffs, New Jersey 07632, 557pp.

Goodger J. Q. D. and Schachtman D. P. (2010). Nitrogen Source Influences Root to Shoot Signaling Under Drought. Abiotic Stress Adaptation in Plants: 165-173.

Krause K, Cherubini P., Bugmann H. and Schleppi P. (2012). Growth enhancement of Picea abies trees under long-term, low-dose $\mathrm{N}$ addition is due to morphological more than to physiological changes. Tree Physiology, 32(12): 1471-1481.

Li J., Yu B., Zhao C., Nowak R. S., Zhao Z., Sheng Y. and Li J. (2013). Physiological and morphological responses of Tamarix ramosissima and Populus euphratica to altered groundwater availability. Tree Physiology, 33 (1): 57-68.

Piper C. S. (1947). Soil and Plant Analysis: 293296. The University of Adelaide, Adelaide.

Pregl F. (1945). Quantitive Organic Micro-analysis $4^{\text {th }}$. Edit., J. \& A. Churchill, Ltd., London.

Rance S. J., Myers R. J. K. and Cameron D. M. (2009). Dynamics of uptake, distribution and utilization of nitrogen applied at different times after planting in a Eucalyptus grandis plantation. Plant and Soil, 324(1-2): 241-252.

Sannappa B., Devaiah M. C., Govindan R. and Prasad N. K. K. (2000). Influence of nitrogenous source fertilizers on yield and foliar constituents of rainfed mulberry. Mysore-Journal-of-Agricultural-Sciences, 34(2): 147-152.

Shvaleva A. L., Costa E., Silva F., Jouve J. E., Hausman J. F., Almeida M. H., Maroco J. P., Rodrigues M. L., Pereira M. M. and Chaves J. S. (2005). Metabolic responses to water deficit in two Eucalyptus globulus clones with contrasting drought sensitivity. Tree Physiology,26(2): 239-248.

Siemens J. A. and Zwiazek J. J. (2013). Effects of nitrate and ammonium on water relations of Trembling Aspen seedlings in solution culture. Journal of Plant Nutrition, 36 (3): 372-389.

Snedecor G.W. and Cochran W.G. (1972). Statistical Methods. The lowa State Univ. Pres., Ames, lowa, U.S.A.

Wang M., Li-Qiu R., Xiao-Dong M. and WangChang K. E. (2004). Effect of water stress on $\mathrm{N} 2 \mathrm{O}$ emission rate of 5 tree species. Journalof-Forestry-Research, 15(1): 19-23.

Warren C. R. and Adams M. A. (2002). Possible causes of slow growth of nitrate-supplied Pinus pinaster. Canadian Journal of Forest Research, 32 (4): 569-580.

Xiao X., Yang F., Zhang S., Korpelainen H. and Li, C. (2009). Physiological and proteomic responses of two contrasting Populus cathayana populations to drought stress. Physiologia Plantarum, 136 (2): 127-249.

Yang Y., Guo J., Wang G., Yang L. and Yang Y. (2012). Effects of drought and nitrogen addition on photosynthetic characteristics and resource allocation of Abies fabri seedlings in eastern Tibetan Plateau. New Forests, 43 (4): 505-518. 
Yang Y., Liu Q. and Wang G. X. (2011). Physiological behaviors of Acer mono under drought and low light. Russian Journal of Plant Physiology, 58(3): 531-537.

Zhao C. and Liu Q. (2009). Growth and physiological responses of Picea asperata seedlings to elevated temperature and to nitrogen fertilization.
Acta Physiologiae Plantarum, 31(1): 163-173. Zheng Y. X., Wu J. C., Cao F. L. and Zhang Y. P. (2010). Effects of water stress on photosynthetic activity, dry mass partitioning and some associated metabolic changes in four provenances of neem (Azadirachta indica A. Juss). Photosynthetica, 48(3): 361369.

$$
\begin{aligned}
& \text { تأثير مصادر النتروجين على النمو والمحتوى الكيميائى لشتيلات السرو } \\
& \text { النامية تحت مستويات مختلفة من السعة العتوى الحقلية } \\
& \text { صفية حمدى الحنفى- هند مصطفى سويفي ـ *عمرو رافت ربيع- *:إيناس حسن محم سليمان } \\
& \text { قسم بساتين الزينة - كلية الزر اعة - جامعة القاهرة- } \\
& \text { * قسم الأشجار الخثبية و الغابات ـ معهد بحوث الزية البساتين - الجيزة } \\
& \text { اجرى هذا البحث بمشتل قسم الاشجار الخشبية بمعهد بحوث البساتين - مركز البحوث الزر اعيةـ الجيزة- وقسم بساتين الزينة }
\end{aligned}
$$

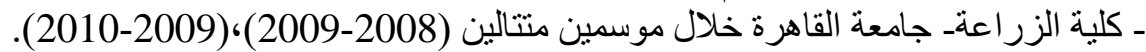

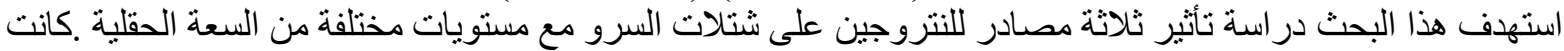

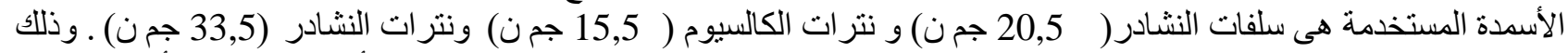

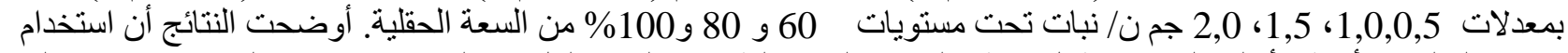

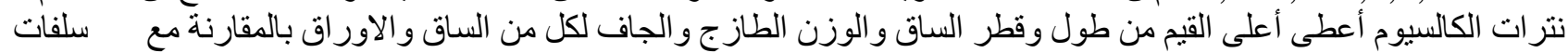

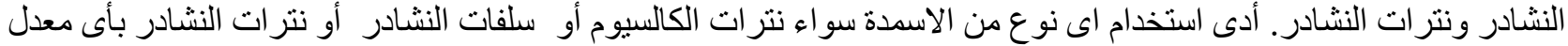

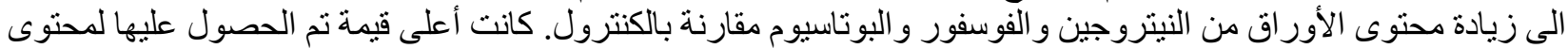

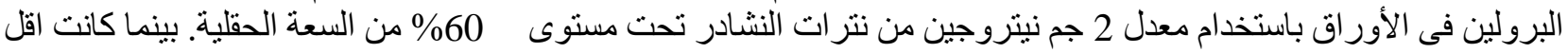

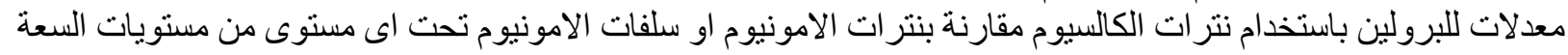

$$
\begin{aligned}
& \text { الحقلية. }
\end{aligned}
$$

المجلة العلمية لكلية الزراعة - جامعة القاهرة - المجلا (64) العدد الثاني ( إبريل 2013): 172-185. 